\title{
Opportunistic Wireless Relay Networks: Diversity-Multiplexing Tradeoff
}

\author{
Mohamed Abouelseoud, Student Member, IEEE, and Aria Nosratinia, Fellow, IEEE
}

\begin{abstract}
The performance of many opportunistic relay networks has been unknown in part because opportunistic analysis relies on independence assumptions that break down in many interesting and useful network topologies. This paper develops techniques that expand opportunistic analysis to a broader class of networks, proposes new opportunistic methods for several network geometries, and analyzes them in the high-SNR regime. For each of the geometries studied in the paper, we analyze the opportunistic DMT of several relay protocols, including amplify-and-forward, decode-and-forward, compress-and-forward, nonorthogonal amplify-forward, and dynamic decode-forward. Among the highlights of the results: In a variety of multi-user single-relay networks, simple selection strategies are developed and shown to be DMT-optimal. It is shown that compress-forward relaying achieves the DMT upper bound in the opportunistic multiple-access relay channel as well as in the opportunistic $n \times n$ user network with relay. Other protocols, e.g., dynamic decode-forward, are shown to be near optimal in several cases. Finite-precision feedback is analyzed for the opportunistic multiple-access relay channel, the opportunistic broadcast relay channel, and the opportunistic gateway channel, and is shown to be almost as good as full channel state information.
\end{abstract}

Index Terms-Cooperative communication, diversity multiplexing trade-off (DMT), opportunistic communication, relay networks.

\section{INTRODUCTION}

$\mathbf{O}$ PPORTUNISTIC communication is a method that at each time chooses the best among multiple communication alternatives in a network. Multiuser diversity [1] is a prominent example: in broadcast channels under quasi-static fading, it is throughput optimal to transmit at each time only to the user with the best channel. Relay selection is another example of opportunistic communication. An early analysis of relay selection without transmit-side channel state information appeared in [2]. Bletsas et al. [3]-[5] investigated amplify-and-forward (AF) relay selection, followed by several other works including [6]-[9]. Decode-and-forward (DF) relay selection has also received attention [10]-[16]. The diversity

Manuscript received November 25, 2009; revised November 04, 2010; accepted March 15, 2011. Date of current version October 07, 2011. This work was supported in part by the National Science Foundation under Grant CNS0435429 and in part by the THECB under Grant 009741-0084-2007.

The authors are with the Department of Electrical Engineering, The University of Texas at Dallas, Richardson, TX 75080 USA (e-mail: m.abolsoud@student.utdallas.edu; aria@utdallas.edu).

Communicated by S. A. Jafar, Associate Editor for Communications.

Color versions of one or more of the figures in this paper are available online at http://ieeexplore.ieee.org.

Digital Object Identifier 10.1109/TIT.2011.2165153 multiplexing tradeoff (DMT) for relay selection has been investigated in a few works including [17] for addressing the multiplexing loss of DF relaying, and [9] for a combination of antenna selection and AF relay selection.

The literature on opportunistic relays, despite its rapid growth, has focused on a relatively restricted set of conditions. Broadly speaking, the scope of previous work has been on geometries and protocols where node selection can be reduced to scalar comparisons of statistically independent link gains (or simple scalar functions thereof). For example, Decode-Forward (DF) relay selection compares the relay-destination links of relays that have decoded the source message. In the case of amplify-forward (AF) relaying, the end-to-end SNR (or a proxy, e.g., in [3]) is used to select relays, which is again a scalar comparison among independent random variables.

This leaves open a significant set of problems for whose analysis the existing approaches are insufficient. Among them one may name even seemingly simple problems, e.g., the DMT of the orthogonal relay on/off problem in the single-relay channel, which has been unsolved until now (see Section IV).

To shed light on the key difficulties, consider the example of the opportunistic multiple-access relay channel (Fig. 1). Two users transmit messages to a common receiver with the assistance of a relay. During each transmission interval either User 1 transmits and User 2 is silent, or vice versa. The goal is to opportunistically choose the user that can access the channel at a higher rate. The main challenge in the analysis of this system is twofold:

1) The selection is a complex function of multiple link gains, i.e., it is not immediately clear how to select the "better" node in an easy and straight forward way. Not only do all the five link gains participate in this decision, but also the capacity of the component relay networks is generally unknown, and even the achievable rates are only known as expressions that involve nontrivial optimizations. Because the performance analysis must take into account the selection function, the complexity of analysis can quickly get out of hand with increasing number of nodes.

2) The relay-destination link is shared among the two opportunistic modes; therefore, the decision variables for the two modes are not statistically independent. The order statistics of dependent random variables are complicated and often not computable in closed form.

One of the contributions of this work is to address or circumvent the above mentioned difficulties. This paper analyzes the diversity and multiplexing gain of a variety of opportunistic relay systems whose asymptotic high-SNR performance has to date been unknown. All networks in this paper have one relay. 


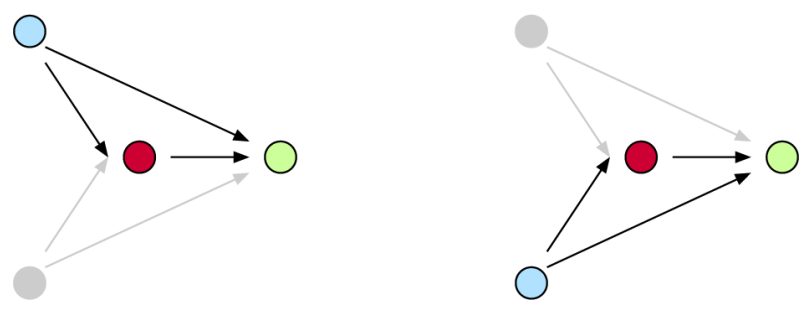

Fig. 1. Opportunistic modes in the multiple-access relay channel.

Among the network geometries that have been studied are the opportunistic multiple-access and broadcast relay channels and several variations of the opportunistic $n \times n$ user network with a relay. In the $n \times n$ network with a relay, if nodes communicate pairwise while crosslink gains cannot be ignored, the links and communication structure resemble an interference channel with a relay; therefore, we call it an opportunistic interference relay channel. ${ }^{1}$ When the crosslink gains can be ignored, we denote it the opportunistic shared relay channel. Finally, if all transmitters have data for all receivers, we denote the scenario as opportunistic X-relay channel. The gateway channel represents a scenario where the only path between sources and destination is through a relay. To summarize, the main results of this paper are as follows:

- To begin with, the DMT of the opportunistic single-relay on/off problem is calculated under DF and AF. This simple result can be used as a building block for the study of larger networks.

- The diversity-multiplexing tradeoff of the opportunistic interference relay channel is calculated under orthogonal $\mathrm{AF}$ and $\mathrm{DF}$, as well as nonorthogonal amplify and forward (NAF), dynamic decode and forward (DDF), and nonorthogonal compress and forward (CF). The nonorthogonal $\mathrm{CF}$ is shown to achieve the DMT upper bound.

- For the shared relay channel, an upper bound for the DMT under opportunistic channel access is calculated. Furthermore, it is shown that for the shared relay channel at low multiplexing gain, the DDF outperforms the NAF and CF while at medium multiplexing gains, the $\mathrm{CF}$ is the best. At high multiplexing gain in the shared relay channel the relay should not be used.

- For the multiple access relay channel, a simple selection scheme based on the source-destination link gains is shown to be optimal for several protocols. Specifically, under this simple selection mechanism, the CF nonorthogonal relaying is shown to achieve the genie-aided DMT upper bound, and the NAF and the DDF also achieve their respective DMT upper bounds (i.e., more intricate selection schemes do not yield a better DMT).

- For the X-relay channel, an opportunistic scheme is presented that meets the DMT upper bound under the CF protocol. For other relaying protocols, the DMT regions are calculated.

\footnotetext{
${ }^{1}$ The naming is for convenience purposes and only reflects the presence of links not the operation of the network. In opportunistic operation there is no interference among users.
}

- The results for the opportunistic broadcast relay channel follow from the opportunistic multiple-access relay channel.

- For the gateway channel, the superposition as well as the orthogonal channel access is studied in the absence of transmit CSI, showing that the latter is almost as good as the former. Then, the opportunistic channel access is fully characterized.

- Finite precision feedback is investigated for the multiple access relay channel (and by implication the broadcast relay channel), as well as the gateway channel. The DMT with finite-precision feedback for several other relay channels remains an open problem.

The organization of the paper is as follows: in Section II, we describe the system model. In Section III, the diversity multiplexing tradeoff for an opportunistic system switches between different access modes is analyzed. In Section IV, the problem of a single-relay opportunistic on/off problem is solved. Then, a succession of DMT analyses is presented for a number of network geometries and relaying protocols: in Section V for the interference relay channel, in Section VI for the shared relay channel, in Section VII for the multiple access relay channel, in Section VIII for the X-relay channel, and in Section IX for the gateway channel. We conclude our work in Section X.

\section{System Model}

All the nodes in the network are single-antenna and due to practical limitations, nodes cannot transmit and receive at the same time (half duplex). The channel between any two nodes experiences flat, quasi-static block fading whose coefficients are known perfectly at the receiver. The opportunistic selection mechanism also has access to channel gains, either in full or quantized. The length of the fading states (coherence length) is such that the source message is transmitted and received within one coherence interval. Furthermore, each transmission can accommodate a codeword of sufficient length so that standard coding arguments apply.

The various networks considered in this paper may have either multiple sources, multiple destinations, or both. In all scenarios in this paper, there is one relay. The channel coefficients between transmitter $i$ and receiver $j$ is denoted with $h_{i j}$. Channel gains to or from a relay are shown with $h_{i r}$ or $h_{r j}$. When the network has only one source, a symbolic index $s$ is used for it; similarly if a network has no more than one destination, the index $d$ will be used for it. For example, in a simple relay channel the links are denoted $h_{s r}, h_{r d}, h_{s d}$. Channel gains are assumed independent identically distributed circularly symmetric complex Gaussian random variables. The received signals are corrupted by additive white Gaussian noise (AWGN) which is $n_{r} \sim \mathcal{C N}(0, N)$ at the relay and $n_{j} \sim \mathcal{C N}\left(0, N_{j}\right)$ at the destinations. Without loss of generality, in the following we assume all noises have unit variance, i.e., $N=N_{j}=1 \forall j$. The transmitter nodes, the sources and the relay, have short-term individual average power constraints for each transmitted codeword. The transmit-equivalent signal-to-noise ratio (SNR) is denoted by $\rho$. Due to the normalization of noise variance, the SNR $\rho$ also serves as a proxy for transmit power. 
In the original definitions of opportunistic communication, e.g., multi-user diversity, only one transmitter is active during each transmission interval. For the relay networks considered in this paper, the definition is slightly generalized in the following manner:

Definition 1: Opportunistic communication is defined as a strategy where the received signal at each node during each transmission interval is independent of all but one of the transmitted messages. In other words, during each transmission interval, each receiver in the network hears only one message stream unencumbered by other message streams. The target message stream may originate from a source, a relay, or both.

This definition maintains the spirit of opportunistic communication while allowing various nonorthogonal relaying strategies. It is noteworthy that with this generalized definition, in some networks (e.g., shared relay channel) more than one message may be in transit at the same time.

Definition 2: An opportunistic communication mode is the set of active transmitters, receivers, and respective links in the network during a given transmission interval.

This work studies the high-SNR behavior of opportunistic relay channels via the diversity-multiplexing tradeoff(DMT), in a manner similar to [18]. Each transmitter $i$ is allocated a family of codes $\mathcal{C}_{i}(\rho)$ indexed by the SNR, $\rho$. The rate $R_{i}(\rho)$ denotes the data rate in bits per second per hertz and is a function of the SNR. The multiplexing gain per user $r_{i}$ is defined as [18]

$$
r_{i}=\lim _{\rho \rightarrow \infty} \frac{R_{i}(\rho)}{\log \rho} .
$$

The selection strategy in the opportunistic relay network yields an effective end-to-end channel. The attempted rate into this effective channel is $R_{i} \approx r_{i} \log \rho$. The error probability subject to this rate is denoted $P_{e}(\rho)$ and the diversity gain is defined as follows:

$$
d=-\lim _{\rho \rightarrow \infty} \frac{\log P_{e}(\rho)}{\log \rho}
$$

For the purposes of this study, since the transmission intervals are sufficiently long, the diversity can be equivalently calculated using the outage probability.

In principle, the high-SNR study of a network can generate a multiplicity of diversities and multiplexing gains. In this paper we pursue the symmetric case, i.e., all opportunistic modes the have the same diversity gain $d$ (in a manner similar to [19]) and also are required to support the same multiplexing gain $r_{i}$, where $r_{i}=r / n$ and $r$ is the overall (sum) multiplexing gain.

Finally a few points regarding notation: The probability of an event is denoted with $\mathbb{P}(\cdot)$. We say two functions $f(x)$ and $g(x)$ are exponentially equal if

$$
\lim _{x \rightarrow \infty} \frac{\log f(x)}{\log g(x)}=1
$$

and denote it with $f(x) \doteq g(x)$. The exponential order of a random variable $X$ with respect to $\operatorname{SNR} \rho$ is defined as

$$
v=-\lim _{\rho \rightarrow \infty} \frac{\log X}{\log \rho}
$$

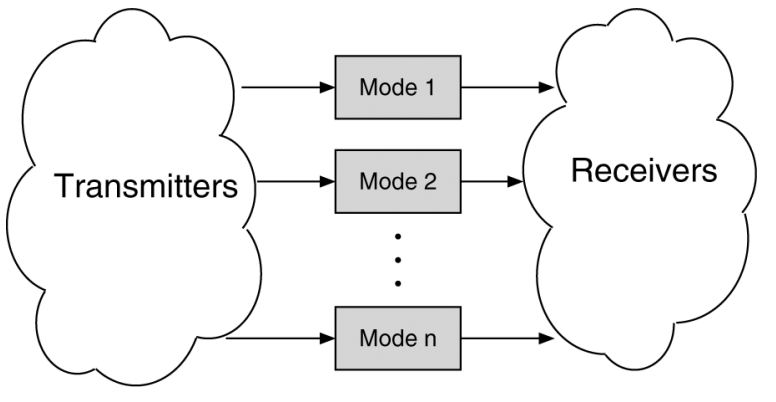

Fig. 2. General opportunistic wireless scenario model. Each mode consists of active links, potentially including a relay.

and denoted by $X \doteq \rho^{-v}, \dot{\leq}$ and $\dot{\geq}$ follow the same definition.

\section{BASIC RESULTS FOR DMT ANALYSIS}

Consider an abstraction of a wireless network, shown in Fig. 2, consisting of a set of sources, a set of destinations, and a number of data-supporting paths between them. Each of these paths may connect one or more source to one or more destination, and may consist of active wireless links as well as (possibly) relay nodes. Recall that each collection of active paths and nodes is called an opportunistic mode. A concrete example of opportunistic modes was shown in Fig. 1, where Source 1, Relay, Destination, and corresponding links make one mode, and Source 2, Relay, Destination, and corresponding links form the second mode. For the purposes of this section, the geometry of the links and relays that compose each mode is abstracted away. However, the DMT supported by each of the modes $^{2}$ is assumed to be known. Furthermore, it is assumed that only one mode can be active at any given time, i.e., we select one mode during each transmission interval.

We now produce a simple but useful result.

Lemma 1: Consider a system that opportunistically switches between $n$ paths (modes) whose conditional DMTs are given by $d_{i}^{\prime}(r)$. The overall DMT is bounded by

$$
d(r) \leq d_{1}^{\prime}(r)+d_{2}^{\prime}(r)+\cdots+d_{n}^{\prime}(r)
$$

where $d_{i}^{\prime}(r)$ is defined as

$$
d_{i}^{\prime}(r)=-\lim _{\rho \rightarrow \infty} \frac{\log \mathbb{P}\left(e_{i} \mid e_{i-1}, \ldots, e_{1}\right)}{\log \rho}
$$

and $\mathbb{P}\left(e_{i} \mid e_{i-1}, \ldots, e_{1}\right)$ is the probability of error in access mode $i$ given that all the previous access modes are in error.

Proof: We demonstrate the result for a two-mode network, generalization for $n$ users follows directly.

The total probability of error when switching between two subsystems is

$$
P_{e}=\mathbb{P}\left(e_{1}, e_{2}\right)+\mathbb{P}\left(U_{1}, e_{1}, e_{2}^{c}\right)+\mathbb{P}\left(U_{2}, e_{1}^{c}, e_{2}\right)
$$

where $e_{1}$ and $e_{2}$ are the events of error in decoding User 1 and User 2 data, respectively, the complements of error events are

\footnotetext{
${ }^{2}$ The multiplexing gain of each mode can be defined as the prelog of the overall rate carried by that mode, and similarly the diversity defined as the slope of the corresponding aggregate error rate of the data.
} 

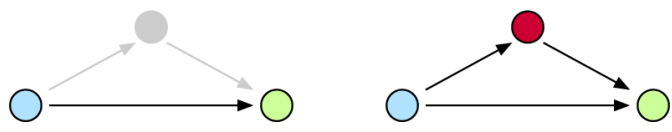

Fig. 3. Opportunistic modes in the simple orthogonal relay channel.

denoted with a superscript $c$, and $U_{1}, U_{2}$ are the events of opportunistically choosing Mode 1 and Mode 2, respectively. The event characterized by the probabilities $\mathbb{P}\left(U_{1}, e_{1}, e_{2}^{c}\right)$ and $\mathbb{P}\left(U_{2}, e_{1}^{c}, e_{2}\right)$ represents the error due to wrong selection.

We can upper bound $P_{e}$ as

$$
\begin{aligned}
P_{e} & \geq \mathbb{P}\left(e_{1}, e_{2}\right) \\
& =\mathbb{P}\left(e_{1}\right) \mathbb{P}\left(e_{2} \mid e_{1}\right) \\
& \doteq \rho^{-d_{1}^{\prime}(r)} \rho^{-d_{2}^{\prime}(r)}
\end{aligned}
$$

which implies that

$$
d(r) \leq d_{1}^{\prime}(r)+d_{2}^{\prime}(r)
$$

where $d_{i}^{\prime}(r)$ is given by (5). This completes the proof.

Specializing Lemma 1 to the case of independent error probabilities directly yields the following.

Lemma 2: A DMT upper bound for opportunistically switching between $n$ independent wireless subsystems is given by $d(r)$ where

$$
d(r) \leq d_{1}(r)+d_{2}(r)+\cdots+d_{n}(r)
$$

and $d_{i}(r)$ is the DMT of the subsystem $i$.

Lemma 3: The upper bounds of Lemma 1 and Lemma 2 are tight if the following two conditions are asymptotically satisfied:

1) Each selected subsystem uses codebooks that achieve its individual DMT.

2) The selection criterion is such that the system is in error only when all subsystems are in error, i.e., $\mathbb{P}\left(U_{1}, e_{1}, e_{2}^{c}\right)=$ $\mathbb{P}\left(U_{2}, e_{1}^{c}, e_{2}\right)=0$.

Throughout the remainder of the paper, we assume that appropriate codebooks are designed and used; therefore, the first condition is satisfied. The second condition would be satisfied by selecting access modes according to their instantaneous end-to-end mutual information. For practical reasons, we may consider simpler selection criteria, in which case the tightness of the bounds above is not automatically guaranteed.

\section{OPPORTUNISTIC ON/OFF RELAY}

In this section we consider a simple orthogonal relaying scenario with one source, one relay and one destination. During each transmission interval, the source transmits during the first half-interval. In the second half-interval, either the relay transmits, or the relay remains silent and the source continues to transmit (see Fig. 3). The decision between these two options is made opportunistically based on the channel gains. ${ }^{3}$

The question is: how should the relay on/off decision be made, and what is the resulting high-SNR performance (DMT).

\footnotetext{
${ }^{3}$ Recall that both half-intervals are within the same coherence interval, i.e., the entire operation observes one set of channel realizations.
}

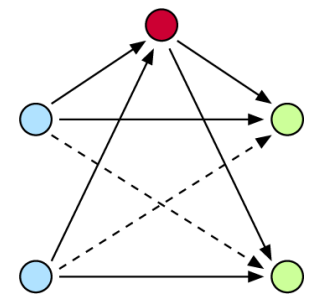

Fig. 4. Interference relay channel.

The apparent simplicity of the problem can be deceiving, because the random variables representing the performances of our two choices are not independent.

Theorem 1: The DMT of a three-node simple relay channel, under either AF or DF, subject to opportunistic relay selection, is given by

$$
d(r)=(1-r)^{+}+(1-2 r)^{+} .
$$

Proof: The proof is relegated to Appendices I and II. An outline of the proof is as follows. The DMT of a point-to-point nonrelayed link is $d(r)=(1-r)^{+}$. DF and AF orthogonal relaying [20] have the DMT $d(r)=(1-2 r)^{+}$. Using the techniques described in the previous section, these two DMTs are combined. The main part of the proof is to establish that the conditional DMT of the relay channel subject to the direct link being in outage is $d(r)=(1-2 r)^{+}$, similar to its unconditional DMT; therefore, the overall result follows from Lemma 1.

Remark 1: For the simple relay channel shown above, there is no need to investigate the opportunistic DDF and NAF, for the following reason. In both NAF and DDF, it can be shown that the end-to-end mutual information is never increased by removing the relay from the network, because channel state information is already incorporated into the operation of NAF and DDF in such a way that the usage of the relay automatically adjusts to the quality of the links.

Remark 2: It has been known that the NAF protocol provides gains over orthogonal AF but the NAF decoding can be complicated due to self-interference. The results of this section show that the DMT gains of the NAF protocol can be achieved with a much simpler decoding by using an opportunistic relay on/off strategy. The cost is a small exchange of channel state information for opportunistic relaying (1-bit feedback from the destination node to the source and the relay).

\section{OPPORTUNISTIC INTERFERENCE RELAY CHANNEL}

This section is dedicated to the study of a $n \times n$ network with a relay in the opportunistic mode. The topology of the links in this network is identical to an interference relay channel; therefore, this structure is called an opportunistic interference relay channel. The naming is a device of convenience inspired by the topology of the network.

For reference purposes, we briefly outline the background of nonopportunistic interference relay channel. The interference channel [21], [22] together with a relay was introduced by Sahin and Erkip [23] (Fig. 4) who present achievable rates using full duplex relaying and rate splitting. Sridharan et al. [24] present an achievable rate region using a combination 


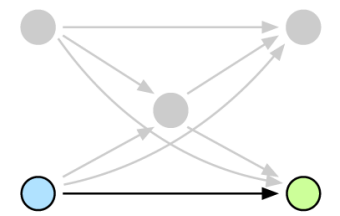

(a)

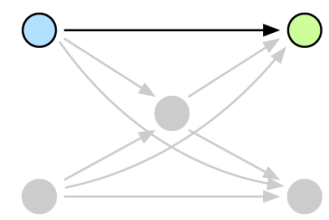

(b)

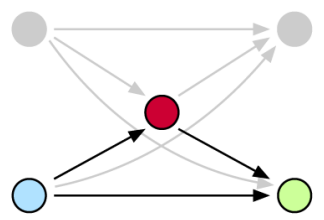

(c)

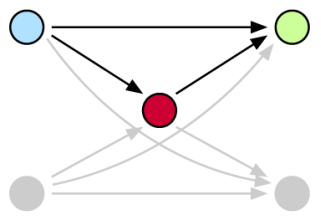

(d)

Fig. 5. Opportunistic access modes for the interference relay channel with orthogonal relaying.

of the Han-Kobayashi coding scheme and Costa's dirty paper coding, and calculate the degrees of freedom. Maric et al. [25] study a special case where the relay can observe the signal from only one source and forward the interference to the other destination. Tannious and Nosratinia [17] show that the degrees of freedom for a MIMO interference relay channel with number of antennas at the relay matching or exceeding the number of users, is $n / 2$ where $n$ is the number of users.

As mentioned earlier, opportunistic modes are defined such that the data streams do not interfere, i.e., each receiving node is exposed to one data stream at a time. Therefore, the twouser interference relay channel has up to four access modes ${ }^{4}$ as shown in Fig. 5. The system selects one of the modes based on the instantaneous link gains. In the following we analyze the network under various relaying protocols and calculate the DMT in each case.

We start by developing a simple genie upper bound. Consider a genie that provides the relay with perfect knowledge of the messages of the transmitting sources. Thus, access modes (c) and (d) are transformed into a MISO channel with a DMT of $2(1-r)^{+}$. If the genie-aided access mode (c) and (d) are in outage, then access modes (a) and (b) will be in outage as well; therefore, they need not be considered. Applying Lemma 1, the DMT of the $2 \times 2$ user opportunistic interference relay channel is upper bounded by $4(1-r)^{+}$. This genie upper bound directly extends to $2 n(1-r)^{+}$for the $n \times n$ user topology.

\section{A. Orthogonal Relaying}

Orthogonal relaying supports the full set of four access modes in Fig. 5. Two of the modes do not involve the relay. In the relay-assisted modes, a source transmits during the first half of the transmission interval and the relay transmits in the second half of the transmission interval.

1) Amplify and Forward Orthogonal Relaying: In the relayassisted modes, the relay amplifies the received signal and forwards it to the destination. We select the mode that minimizes the outage probability. The instantaneous mutual information of the nonrelay access modes is given by $I_{i}=\log \left(1+\left|h_{i i}\right|^{2} \rho\right)$ where $i=1.2$. The instantaneous mutual information for the relay-assisted modes under orthogonal AF is given by [20], [26]

$I_{i+2}=\frac{1}{2} \log \left(1+\left|h_{i i}\right|^{2} \rho+f\left(\left|h_{i r}\right|^{2} \rho,\left|h_{r i}\right|^{2} \rho\right)\right), i=1,2$

where $f(x, y)=\frac{x y}{x+y+1}$. The selection criterion is as follows. We first check the direct links. If none of the direct links can

\footnotetext{
${ }^{4}$ In nonorthogonal $\mathrm{CF}, \mathrm{DDF}$, and NAF relaying protocols, the nonrelayed modes never support higher rates than the relayed modes. Therefore, in CF, DDF, NAF some of these modes are never selected and can be ignored.
}

support the rate $r \log \rho$, we check the access modes (c) and (d). Using Lemma 1, the total DMT is given by

$$
d(r)=d_{1}^{\prime}(r)+d_{2}^{\prime}(r)+d_{3}^{\prime}(r)+d_{4}^{\prime}(r)
$$

where

$$
\begin{aligned}
& d_{1}^{\prime}(r)=\lim _{\rho \rightarrow \infty} \frac{\log \mathbb{P}\left(e_{1}\right)}{\log \rho}, \quad d_{2}^{\prime}(r)=\lim _{\rho \rightarrow \infty} \frac{\log \mathbb{P}\left(e_{2}\right)}{\log \rho} \\
& d_{3}^{\prime}(r)=\lim _{\rho \rightarrow \infty} \frac{\log \mathbb{P}\left(e_{3} \mid e_{1}\right)}{\log \rho}, \quad d_{4}^{\prime}(r)=\lim _{\rho \rightarrow \infty} \frac{\log \mathbb{P}\left(e_{4} \mid e_{2}\right)}{\log \rho}
\end{aligned}
$$

It is easy to verify that $e_{1}$ and $e_{3}$ are independent from $e_{2}$ and $e_{4}$. Using techniques similar to the proof of Theorem 1, the outage probability of the opportunistic orthogonal AF $2 \times 2$ interference relay channel at high SNR is given by

$$
\begin{aligned}
\mathbb{P}(I<r \log \rho) \approx & \left(\frac{e^{-2 \rho^{2 r-1}}-e^{-\rho^{r-1}}-e^{-2 \rho^{2 r-1}+\rho^{r-1}}+1}{1-e^{-\rho^{r-1}}}\right)^{2} \\
& \times\left(1-e^{-\rho^{r-1}}\right)^{2}
\end{aligned}
$$

The total DMT can be shown to be

$$
d(r)=2(1-r)^{+}+2(1-2 r)^{+} .
$$

Generalization to $n$ source-destination pairs follows easily; the corresponding DMT is $d(r)=n(1-r)^{+}+n(1-2 r)^{+}$.

2) Decode and Forward Orthogonal Relaying: We use the same selection technique used in the orthogonal AF relaying. The instantaneous mutual information for the relay-assisted modes is given by by [20]

$$
I_{i+2}=\frac{1}{2} \log \left(1+\rho U_{i}\right), \quad i=1,2
$$

where

$$
U_{i}= \begin{cases}2\left|h_{i i}\right|^{2} & \left|h_{i r}\right|^{2}<\frac{\rho^{2 r}-1}{\rho} \\ \left|h_{i i}\right|^{2}+\left|h_{r i}\right|^{2} & \left|h_{i r}\right|^{2} \geq \frac{\rho^{2 r}-1}{\rho}\end{cases}
$$

With the same type of argument used to calculate the DMT for the opportunistic orthogonal AF interference relay channel and Appendix I, the outage probability of the opportunistic orthogonal $2 \times 2$ DF interference relay channel at high SNR is given by

$$
\begin{aligned}
\mathbb{P}(I<r \log \rho) \approx & \left(1-e^{-\rho^{2 r-1}}\right. \\
& \left.+\frac{\left(1-e^{-\rho^{r-1}}-\rho^{r-1} e^{-\rho^{2 r-1}}\right) e^{-\rho^{2 r-1}}}{1-e^{-\rho^{r-1}}}\right)^{2} \\
& \times\left(1-e^{-\rho^{r-1}}\right)^{2}
\end{aligned}
$$


It can be shown that the DMT in case of orthogonal DF is

$$
d(r)=n(1-r)^{+}+n(1-2 r)^{+} .
$$

\section{B. Nonorthogonal Relaying}

In the nonorthogonal protocols considered in this section, the source transmits throughout the transmission interval, while the relay transmits during part of the transmission interval. The source and relay signals are superimposed at the destination. Note that this superposition does not violate our working definition of opportunistic communication, which states that the received signal at each destination is independent of all but one of the transmitted messages.

Under the nonorthogonal relaying protocols, the interference relay channel has only two access modes, Fig. 5(c) and (d). Access modes (a) and (b) are not considered, because it can be shown that in nonorthogonal relaying, the end-to-end mutual information of the relay-assisted modes is always greater than the corresponding nonrelayed modes.

1) Nonorthogonal Amplify and Forward: For half the transmission interval, the received signal at the destination and at the relay are given by [27]

$$
y_{1 i}=\sqrt{\rho} h_{i i} x_{1 i}+n_{1 i}, \quad y_{1 r}=\sqrt{\rho} h_{i r} x_{1 i}+n_{1 r}
$$

The variables $x, y, n$ have two subscripts indicating the appropriate half-interval and node identity, respectively. For example, $y_{1 r}$ is the received signal during the first half-interval at the relay, while $x_{1 i}$ is the transmit signal at the first half-interval from source $i$. At the second half of the transmission interval the relay normalizes the received signal (to satisfy the relay power constraint) and retransmits it. The destination received signal in the second half is given by

$$
y_{2 i}=\sqrt{\rho} h_{i i} x_{2 i}+\frac{\sqrt{\rho} h_{r i}}{\sqrt{\rho\left|h_{i r}\right|^{2}+1}} y_{1 r}+n_{2 i}
$$

where a similar notation holds. The effective destination noise during this time is $\frac{\sqrt{\rho} h_{r i}}{\sqrt{\rho\left|h_{i r}\right|^{2}+1}} n_{1 r}+n_{2 i}$.

User $i^{*}$ is selected to maximize the mutual information, which at high SNR can be shown to lead to the following selection rule:

$$
i^{*}=\arg \max _{i} I_{i}=\arg \max _{i}\left\{\frac{\left|h_{i i}\right|^{4}\left|h_{i r}\right|^{2}}{\left|h_{r i}\right|^{2}+\left|h_{i r}\right|^{2}}\right\} .
$$

Using our knowledge of the DMT of nonopportunistic NAF [28] which is given by $d(r)=(1-r)^{+}+(1-2 r)^{+}$, and applying Lemmas 2, 3 and using the selection criterion $i^{*}$ from (17), the DMT of opportunistic NAF interference relay channel with $n$ source-destination pairs is

$$
d(r)=n(1-r)^{+}+n(1-2 r)^{+} .
$$

2) Dynamic Decode and Forward: The relay listens to the source until it has enough information to decode. The relay re-encodes the message using an independent Gaussian codebook and transmits it during the remainder of the transmission interval. The time needed for the relay to decode the message depends on the quality of the source-relay channel. Using [28] and Lemma 1, the DMT of the optimal opportunistic DDF interference relay channel is as follows:

$$
d(r)= \begin{cases}2 n(1-r), & 0 \leq r \leq \frac{1}{2} \\ n \frac{1-r}{r}, & \frac{1}{2}<r \leq 1\end{cases}
$$

Compared to the other protocols considered for the interference relay channel, the DDF mutual information for each node has a more complex expression. This provides an impetus for the analysis of simpler selection scenarios. It has been observed elsewhere in this paper that selection based on source-destination link gains sometimes may perform well; therefore, we consider that choice function for the DDF interference relay channel. Following the same technique as [29], the resulting DMT can be shown to be

$$
d(r)= \begin{cases}(n+1)(1-r), & 0 \leq r<\frac{n}{n+1} \\ n \frac{1-r}{r}, & \frac{n}{n+1} \leq r \leq 1\end{cases}
$$

It is observed that for DDF, selection based on direct link gains is clearly suboptimal, especially at low multiplexing gains.

3) Compress and Forward: Following [30], the relay listens to the selected source for a percentage $t$ of the transmission interval. The source and the relay perform block Markov superposition coding, and the destination employs backward decoding [31]. The relay performs Wyner-Ziv compression, exploiting the destination's side information. This ensures that the relay message can be received error free at the receiver. The relay compression ratio must satisfy

$$
I\left(\hat{y}_{r} ; y_{r} \mid x_{r}, y_{d}\right) \leq I\left(x_{r} ; y_{d}\right) .
$$

Yuksel and Erkip [30] show that the optimal DMT, $d(r)=$ $2(1-r)^{+}$, is achieved when the relay listens for half the transmission interval and transmits during the remainder of time in the interval. ${ }^{5}$

For opportunistic compress and forward interference relay channel, the user $i^{*}=\arg \max _{i} I_{i}$ is selected, where $I_{i}$ is the mutual information for each access mode. At high-SNR, using results from [30], the selected user $i^{*}$ can be proved to be

$$
i^{*}=\arg \max _{i} \frac{\left(\left|h_{s_{i}, r}\right|^{2}+\left|h_{s_{i}, d_{i}}\right|^{2}\right)\left(\left|h_{r, d_{i}}\right|^{2}+\left|h_{s_{i}, d_{i}}\right|^{2}\right)\left|h_{s_{i}, d_{i}}\right|^{2}}{\left(\left|h_{s_{i}, r}\right|^{2}+\left|h_{s_{i}, d_{i}}\right|^{2}\right)+\left(\left|h_{r, d_{i}}\right|^{2}+\left|h_{s_{i}, d_{i}}\right|^{2}\right)} .
$$

Each mode can achieve a DMT $d(r)=2(1-r)^{+}$; hence, the opportunistic system with $n$ source-destination pairs can achieve the DMT $d(r)=2 n(1-r)^{+}$.

Fig. 6 compares the DMT of various relaying schemes for the interference relay channel with four source-destination pairs. The optimal opportunistic DDF relaying is denoted by DDF1 and DDF relaying with the simple selection criterion (based on source-destination link gains) is denoted by DDF2. Compress

\footnotetext{
${ }^{5}$ The work in [30] assumes transmit channel state information at the relay to insure that the relay's message reaches the destination error free. Recent work [32] proves that the same DMT can be achieved using quantize-and-map relaying with only receiver-side channel state information. Another relaying protocol, dynamic compress-and-forward, is analyzed in [33] without a direct link and is shown to achieve the optimal DMT without channel state information at the relay.
} 


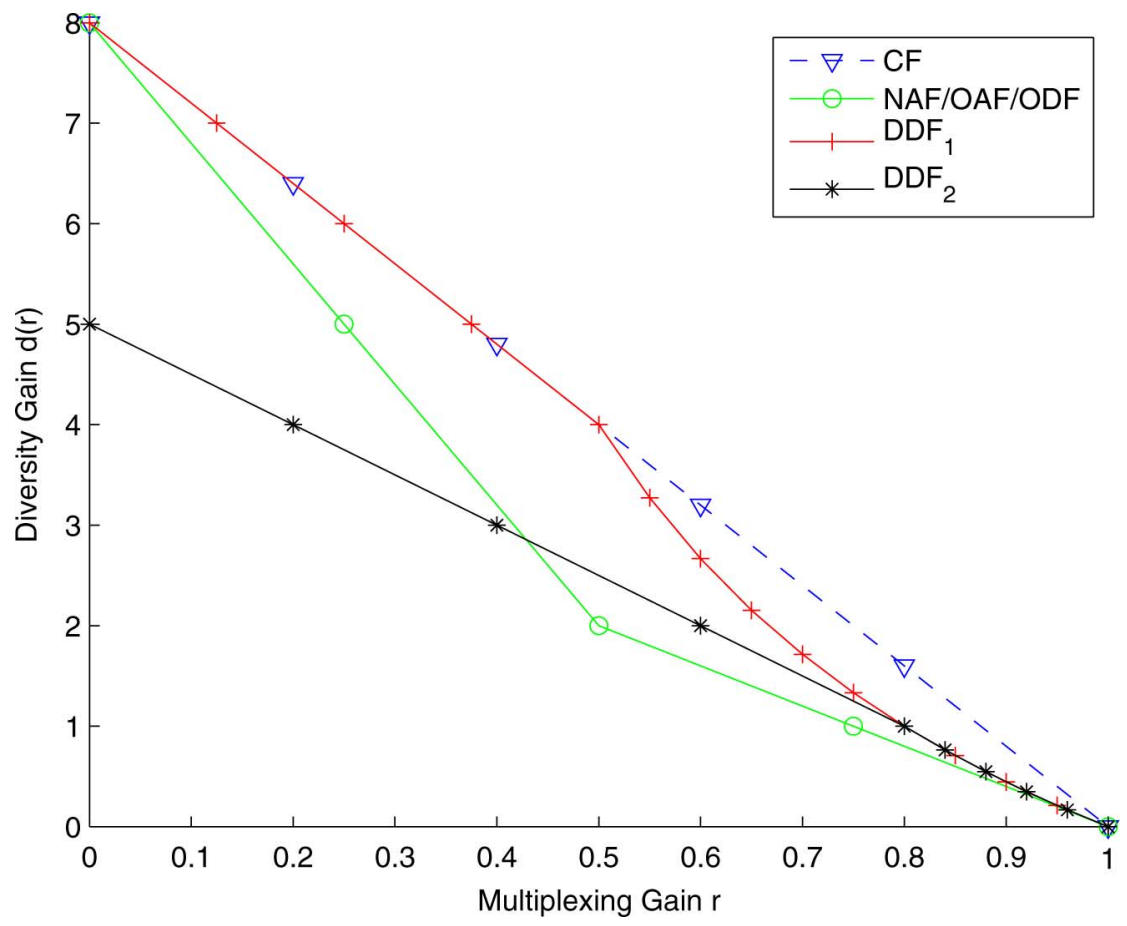

Fig. 6. Diversity multiplexing trade-off for a 4 source-destination pairs interference relay channel using different opportunistic relaying schemes.

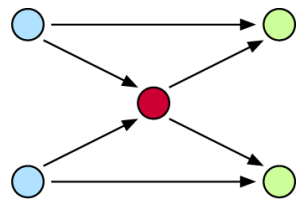

Fig. 7. Shared relay channel.

and forward achieves the optimal DMT but requires full CSI at the relay.

\section{OpPortunistic Shared Relay ChanNel}

The shared relay channel (SRC) (Fig. 7) was introduced in [34] with the sources using TDMA channel access and orthogonal source and relay transmissions. In [35], based on superposition and dirty paper coding, lower and upper bounds on the capacity of additive white Gaussian noise (AWGN) MIMO shared relay channel are presented.

In the shared relay channel, the direct link between each source and its destination is free from interference from the other source; however, the relay can cause indirect interference if it assists both sources at the same time. Therefore, in the opportunistic mode the relay should either assist one of the users or none of them (Fig. 8). We assume the access mode that minimizes the outage probability is chosen. In our analyses, access modes support equal rate; thus, in the first two access modes, one source transmits at rate $R=r \log \rho$, while in the third access mode both sources transmit, each with a rate $R_{i}=r / 2 \log \rho$.

\section{A. DMT Upper Bound}

An easy upper bound can be found by adding the DMT of the three access modes without considering the dependencies

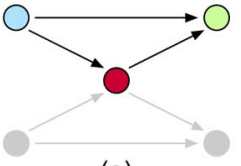

(a)

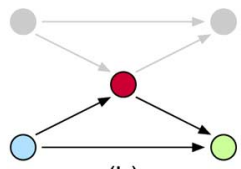

(b)

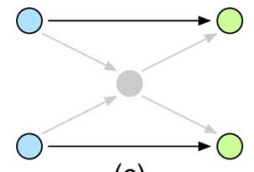

(c)
Fig. 8. Opportunistic access modes for the shared relay channel.

among the throughputs of the three access modes. A tighter upper bound can be found by assuming a genie that provides the relay with the source information. In Fig. 8, we call modes (a) and (b) relay-assisted access modes while denoting mode (c) a nonrelayed access mode. Thus, in the presence of a genie, the relay-assisted access modes are essentially equivalent to MISO links. The nonrelay access mode is obviously not affected by the genie.

Theorem 2: A DMT upper bound for the genie aided opportunistic shared relay channel is given by

$$
\begin{aligned}
d(r) & \leq\left(1-\frac{r}{n}\right)^{+}+(2 n-1)(1-r)^{+} \\
& = \begin{cases}2 n-\left(2 n-1+\frac{1}{n}\right) r, & 0 \leq r \leq 1 \\
\left(1-\frac{r}{n}\right), & 1<r \leq n .\end{cases}
\end{aligned}
$$

Proof: The proof uses Lemma 1 taking into account the dependency between the different access modes. Details of the proof are given in Appendix III.

We notice that for high multiplexing gain, $r>1$, the first and second access modes do not contribute to the diversity gain because the third mode is always active. For low multiplexing gain, $r \leq 1$, the three access modes are contributing to the total diversity gain of the system and switching between the three access modes should be considered. 
For clarity of exposition, we assume two source-destination pairs in the remainder of the analysis. However, the analysis is extendable to any number of node pairs in a straightforward manner.

\section{B. Achievable DMT}

If we allow ourselves to be guided by the upper bound above, it is reasonable to use the nonrelay access mode for high multiplexing gains $(r>1)$. This makes intuitive sense, since relayed access modes cannot support high multiplexing gains. For multiplexing gains less than 1 , switching between the three access mode should be considered.

To begin with, we consider a simplified selection by partitioning the decision space: in one partition (at low multiplexing gains) choosing only among relayed access modes (easier due to their independence), in the other partition (at high multiplexing gains) using only the nonrelayed mode. This hybrid scheme avoids the cost of the comparison among all three modes which is especially useful in the cases of large number of users. Using results from [28] and [30], this strategy leads to the following DMT for NAF $d(r)=\max \left\{2(1-r)^{+}+2(1-2 r)^{+},\left(1-\frac{r}{2}\right)^{+}\right\}$, for DDF

$$
d(r)= \begin{cases}4(1-r), & 0 \leq r \leq 0.5 \\ 2 \frac{1-r}{r}, & 0.5<r \leq 3-\sqrt{5} \\ 1-\frac{r}{2}, & 3-\sqrt{5}<r \leq 2 .\end{cases}
$$

and for CF $d(r)=\max \left\{4(1-r)^{+},\left(1-\frac{r}{2}\right)^{+}\right\}$.

Naturally, there is no guarantee that the above strategy is optimal. For the best results, one must compare directly the three opportunistic modes, but then the DMT requires nontrivial calculations, as characterized by the following results.

The following DMT are subject to the two conditions mentioned in Lemma 3.

\section{1) Nonorthogonal Amplify and Forward:}

Theorem 3: The overall DMT for the opportunistic shared relay channel under NAF relaying protocol is given by

$$
\begin{aligned}
d(r) & =2(1-2 r)^{+}+\left(1-\frac{r}{2}\right)^{+}+(1-r)^{+} \\
& = \begin{cases}4-\frac{11}{2} r, & 0 \leq r \leq 0.5 \\
2-\frac{3}{2} r, & 0.5<r \leq 1 \\
1-\frac{r}{2}, & 1<r \leq 2 .\end{cases}
\end{aligned}
$$

Proof: The proof uses Lemma 1 and results from MIMO point to point communication [18] and NAF relaying [28] taking into account the dependency between the different access modes. Details are given in Appendix IV.

\section{2) Dynamic Decode and Forward:}

Theorem 4: The overall DMT for the opportunistic shared relay channel under DDF relaying protocol is given by (26), shown at the bottom of the page.

Proof: The proof uses Lemma 1 and results for DDF relaying [28], while taking into account the dependency between the three access modes. Details are given in Appendix VI.

3) Compress and Forward: As mentioned earlier, the hybrid strategy yields the following DMT:

$$
d(r)= \begin{cases}4(1-r), & 0 \leq r \leq \frac{6}{7} \\ \left(1-\frac{r}{2}\right), & \frac{6}{7}<r \leq 2 .\end{cases}
$$

One can show that optimization between all three access modes at each $r$ cannot yield a better DMT under CF relaying; therefore, the result above cannot be improved upon. The proof is given in Appendix VII.

Remark 3: The trivial hybrid scheme of using the relay assisted modes at low multiplexing gains and the direct links at high multiplexing links is not always suboptimal. It is shown that for NAF and DDF, better performance is achieved by considering the three access modes at low multiplexing gains. For CF relaying, the nonrelayed access mode is not helping at low multiplexing gains; hence, the hybrid scheme is optimal.

Remark 4: Using the same technique used to prove the DMT of the orthogonal opportunistic simple relay channel, Appendix I and II, and Lemma 1, one can show that the DMT of the opportunistic shared relay channel under either orthogonal $\mathrm{AF}$ or orthogonal $\mathrm{DF}$ is given by

$$
d(r)=2(1-2 r)^{+}+(1-r / 2)^{+}
$$

where the access modes are defined as before and the relay always transmits orthogonal to the sources.

To summarize the results for the opportunistic shared relay channel, a brief comparison between three relaying protocols $\mathrm{NAF}, \mathrm{DDF}$, and $\mathrm{CF}$ is as follows. At low multiplexing gain the DDF outperforms NAF and CF. At medium multiplexing gains, the relay does not have enough time to fully forward the decoded message to the destination and the CF in this case outperforms the DDF. At multiplexing gains above 1, it does not matter which relaying protocol is used since the DMT-optimal strategy uses direct (nonrelayed) mode.

$$
d(r)= \begin{cases}\left(1-\frac{r}{1-r}\left(1-\frac{r}{2}\right)\right)+2(1-r)+\left(1-\frac{r}{2}\right), & 0 \leq r \leq 0.5 \\ 2 \frac{(1-r)}{r}, & 0.5<r \leq 2-\sqrt{2} \\ \frac{(1-r)}{r}+\left(1-\frac{r}{2}\right), & 2-\sqrt{2}<r \leq 1 \\ \left(1-\frac{r}{2}\right), & 1<r \leq 2\end{cases}
$$




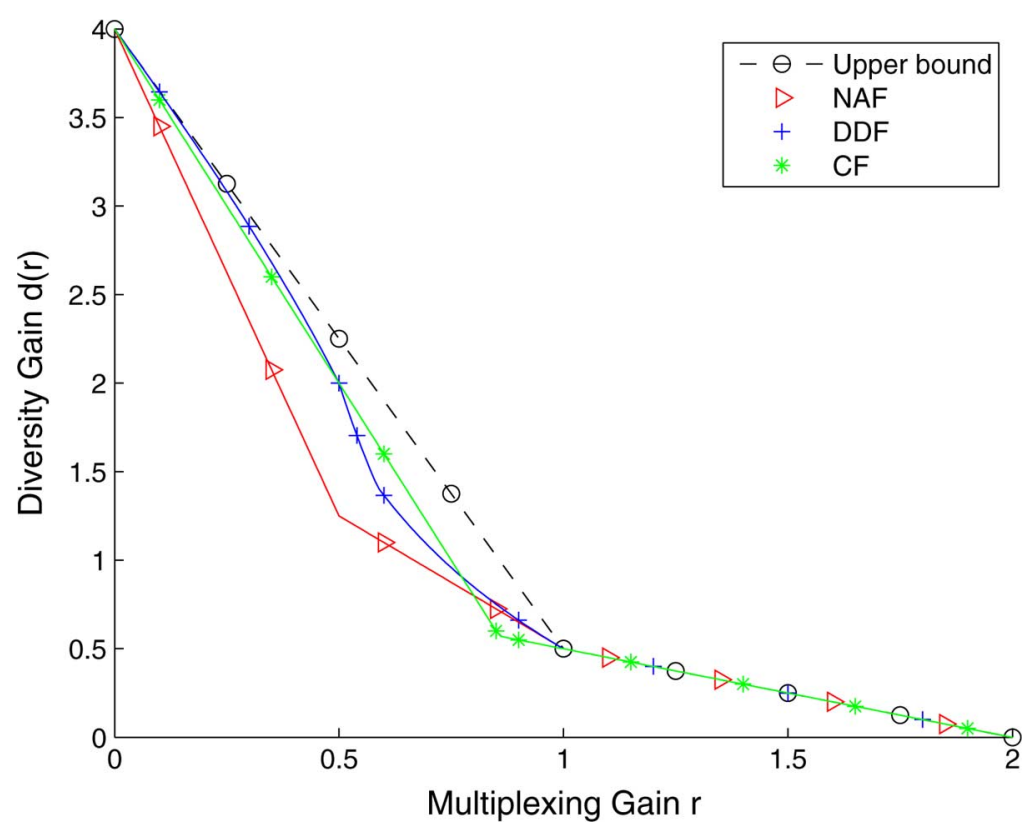

Fig. 9. Diversity multiplexing trade-off for a 2-pair shared relay channel, demonstrating the performance of various protocols.

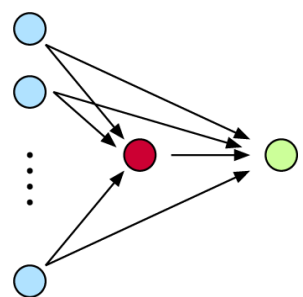

Fig. 10. Multiple access relay channel.

\section{Opportunistic Multiple Access AND BROADCAST RELAY CHANNELS}

The multiple access relay channel (MARC) [36] consists of the standard multiple access channel together with one relay (see Fig. 10). No results for the DMT of the opportunistic MARC have been available until now, but its nonopportunistic DMT under superposition coding with single-antenna nodes is analyzed in [37]-[39], [30]. The following results are known for the nonopportunistic MARC: It is known that the dynamic decode and forward is DMT optimal for low multiplexing gain [37]. The compress and forward protocol achieves a significant portion of the half duplex DMT upper bound for high multiplexing gain [30] but suffers from diversity loss in the low multiplexing regime. The multiple-access relay amplify and forward (MAF) is proposed in [39], it dominates the CF and outperform the DDF protocol in high multiplexing regime.

The broadcast relay channel (BRC) was introduced independently in [40] and [41]. Assuming single-antenna nodes, the opportunistic BRC is identical to the opportunistic MARC except for certain practicalities in the exchange of channel state information, which does not make a difference at the abstraction level of the models used in this paper. Therefore, for the demonstration purposes we focus on MARC; the results carry over to the BRC directly.

\section{A. DMT Upper Bound}

In order to calculate a DMT upper bound for the opportunistic MARC, we assume a genie gives the relay an error-free version of the messages originating from all the sources. We also assume full cooperation on the transmit side. Under these conditions, the source that maximizes the instantaneous end-to-end mutual information is selected. Each of the $n$ sources has an independent link to the destination and they all share the same relay-destination link. The opportunistic modes are demonstrated in Fig. 11. The genie-aided MARC is equivalent to a MISO system with $n+1$ transmit antennas and one receive antenna.

The performance of the opportunistic genie-aided MARC is, therefore, upper bounded by a $(n+1) \times 1$ MISO system with antenna selection that chooses for each codeword transmission two transmit antennas. The $(n+1) \times 1$ antenna selection allows configurations that do not have a counterpart in the opportunistic modes in the MARC channel; therefore, due to the extra flexibility, the MISO system with antenna selection upper bounds the performance of the genie-aided opportunistic MARC channel.

The DMT of a $M \times N$ MIMO link with $L_{t}<M$ selected transmit antennas and $L_{r}<N$ selected receive antennas is upper bounded by a piecewise linear function obtained by connecting the following $K+2$ points [42]

$$
\left\{\left(n,\left(M_{r}-n\right)\left(M_{t}-n\right)\right)\right\}_{n=0}^{K}, \quad\left(\min \left(L_{r}, L_{t}\right), 0\right)
$$

where

$$
\begin{aligned}
K & =\arg \min _{k \in \mathbb{Z}} \frac{\left(M_{r}-k\right)\left(M_{t}-k\right)}{\min \left(L_{r}, L_{t}\right)-k} \\
& \text { subject to } 0 \leq k \leq \min \left(L_{r}, L_{t}\right)-1 .
\end{aligned}
$$



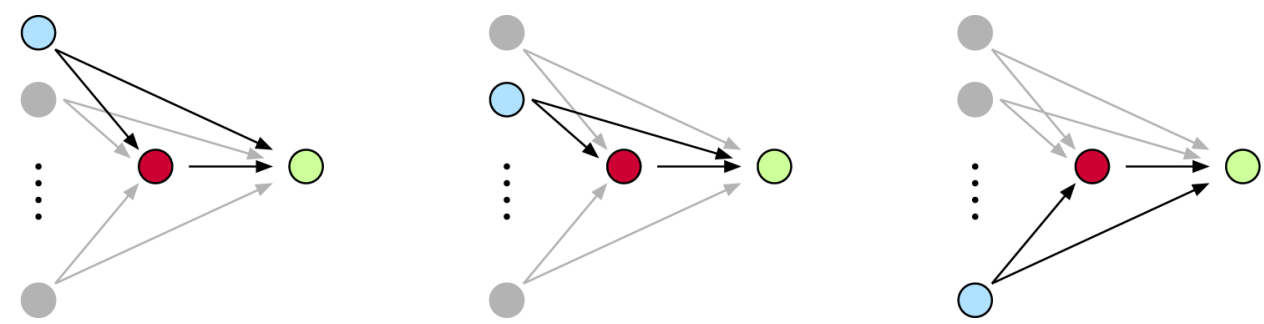

Fig. 11. Opportunistic access modes for the genie-aided multiple access relay channel.

Using this result, a $(n+1) \times 1$ MISO system with two selected transmit antennas has a DMT that is upper bounded by

$$
d(r)=(n+1)(1-r)^{+} .
$$

This represents our genie-aided upper bound for opportunistic MARC

\section{B. Achievable DMT}

In this section, we propose a node selection rule and calculate the corresponding achievability results for a number of relaying protocols in opportunistic MARC and BRC. As mentioned earlier, one of the difficulties in the computation of DMT in opportunistic scenarios is the dependencies among the statistics of the node selections, which itself is a result of selection rules. To circumvent these difficulties, we propose a selection rule that relies only on the source-destination links in the MARC. Because this method does not observe the shared link in the system, the resulting node statistics are independent and many of the computational difficulties disappear.

We shall see that this simplified selection works surprisingly well in the high-SNR regime. It will be shown that for some relaying protocols this selection algorithm yields achievable DMT that is tight against the upper bound.

The proposed schemes for the MARC can also be used for the $\mathrm{BRC}$; therefore, for demonstration purposes, we limit ourselves to MARC. The only difference is that for the BRC the CSI must be fed back to the source to make the scheduling decision.

1) Orthogonal Amplify and Forward: The maximum instantaneous mutual information between the inputs and the output is

$$
I_{A F}=\frac{1}{2} \log \left(1+\rho\left|h_{i^{*} d}\right|^{2}+f\left(\rho\left|h_{i^{*} r}\right|^{2}, \rho\left|h_{r d}\right|^{2}\right)\right)
$$

where $i^{*}=\arg \max _{i}\left|h_{i d}\right|$. The outage probability is given by

$$
\begin{aligned}
P_{A F} & =\mathbb{P}\left(I_{A F}<r \log \rho\right) \\
& =\mathbb{P}\left(\left|h_{i^{*} d}\right|^{2}+\frac{1}{\rho} f\left(\rho\left|h_{i^{*} r}\right|^{2}, \rho\left|h_{r d}\right|^{2}\right)<\frac{\rho^{2 r}-1}{\rho}\right) .
\end{aligned}
$$

Since channel coefficients $h_{i j}$ are complex Gaussian, $\left|h_{i j}\right|^{2}$ obey exponential distributions. We, therefore, use the following result to characterize (32) in the high-SNR regime.
Lemma 4: Assume random variables $u_{i}, v$ and $w$ obey exponential distributions with parameters $\lambda_{u}, \lambda_{v}$ and $\lambda_{w}$, respectively, and $\epsilon$ is a constant and $f(x, y)=\frac{x y}{x+y+1}$

$$
\begin{gathered}
\lim _{\rho \rightarrow \infty} \frac{1}{\left(\frac{\rho^{2 r}-1}{\rho}\right)} \mathbb{P}\left(u_{i}<\frac{\rho^{2 r}-1}{\rho}\right)=\lambda_{u} \\
\lim _{\rho \rightarrow \infty} \frac{1}{\left(\frac{\rho^{2 r}-1}{\rho}\right)^{n} \mathbb{P}\left(\max _{i} u_{i}<\frac{\rho^{2 r}-1}{\rho}\right)=\lambda_{u}^{n}} \\
\lim _{\rho \rightarrow \infty} \frac{1}{\left(\frac{\rho^{2 r}-1}{\rho}\right)^{n+1}} \mathbb{P}\left(\max _{i} u_{i}+v<\frac{\rho^{2 r}-1}{\rho}\right)=\frac{\lambda_{v} \lambda_{u}^{n}}{n+1} \\
\lim _{\rho \rightarrow \infty} \frac{1}{\left(\frac{\rho^{2 r}-1}{\rho}\right)^{n+1}} \mathbb{P}\left(\max _{i} u_{i}+f\left(\frac{v}{\epsilon}, \frac{w}{\epsilon}\right)<\frac{\rho^{2 r}-1}{\rho}\right) \\
=\frac{\lambda_{u}^{n}\left(\lambda_{v}+\lambda_{w}\right)}{2}
\end{gathered}
$$

Proof: Expression (33) is proved in [20]. The proof of the other expressions is similar (with slight modifications) and is omitted for brevity.

From (32) and (36), the probability of outage at high SNR is

$$
P_{A F} \doteq \frac{1}{2} \lambda_{i^{*} d}^{n}\left(\lambda_{i^{*} r}+\lambda_{r d}\right)\left(\frac{\rho^{2 r}-1}{\rho}\right)^{n+1}
$$

where $\lambda_{i^{*} r}, \lambda_{r d}, \lambda_{i^{*} d}$ are the exponential parameters of the channel gains for the links corresponding to the selected opportunistic mode. It follows that the DMT of the opportunistic $n$-user MARC with orthogonal amplify-and-forward, under a selection rule based on the source-destination channel gain, is given by

$$
d(r)=(n+1)(1-2 r)^{+}
$$

2) Orthogonal Decode and Forward: With the orthogonal DF protocol, outage happens if either of the following two scenarios happen: (1) the relay cannot decode and the direct sourcedestination channel is in outage, or (2) the relay can decode but 
the source-destination and relay-destination links together are not strong enough to support the required rate. In other words

$$
\begin{aligned}
& P_{D F}= \mathbb{P} \\
&\left(\left|h_{i^{*} r}\right|^{2} \geq \frac{\rho^{2 r}-1}{\rho}\right) \mathbb{P}\left(\left|h_{i^{*} d}\right|^{2}+\left|h_{r d}\right|^{2}<\frac{\rho^{2 r}-1}{\rho}\right) \\
& \quad+\mathbb{P}\left(\left|h_{i^{*} r}\right|^{2}<\frac{\rho^{2 r}-1}{\rho}\right) \mathbb{P}\left(\left|h_{i^{*} d}\right|^{2}<\frac{\rho^{2 r}-1}{\rho}\right) \\
& \doteq \mathbb{P}\left(\left|h_{i^{*} r}\right|^{2} \geq \rho^{2 r-1}\right) \mathbb{P}\left(\left|h_{i^{*} d}\right|^{2}+\left|h_{r d}\right|^{2}<\rho^{2 r-1}\right) \\
& \quad+\mathbb{P}\left(\left|h_{i^{*} r}\right|^{2}<\rho^{2 r-1}\right) \mathbb{P}\left(\left|h_{i^{*} d}\right|^{2}<\rho^{2 r-1}\right) .
\end{aligned}
$$

Using Lemma 4, specifically (33), (34), and (35), the outage probability can be approximated, thus

$$
P_{D F} \doteq\left(\frac{\lambda_{i^{*} d}^{n} \lambda_{r d}}{n+1}+\lambda_{i^{*} d}^{n} \lambda_{r d}\right) \rho^{(n+1)(2 r-1)} .
$$

It follows directly that the $n$-user opportunistic MARC, subject to selection based on source-destination channel gains and operating with orthogonal DF, has the following DMT

$$
d(r)=(n+1)(1-2 r)^{+}
$$

Remark 5: We know that an orthogonal relay may not be helpful in high multiplexing gains, but the above orthogonal MARC dedicates time to the relay; therefore, it may be improved. To do that, we add to the system $n$ unassisted modes, where the relay does not play a role. For an opportunistic MARC that can choose between $2 n$ opportunistic modes, one can show that the maximum achieved DMT is $d(r)=n(1-r)^{+}+(1-$ $r / 2)^{+}$. A simple selection rule achieves this DMT: take the best source-destination link. If it is viable without the relay, use it without relay, otherwise use it with the relay.

3) Nonorthogonal Amplify and Forward: In this protocol, the source with the maximum source-destination channel coefficient is selected. Recall that the index of this source is denoted $i^{*}$. This source continues transmitting throughout the transmission interval.

The DMT of the MARC with $\mathrm{n}$ sources and opportunistic channel access based on the source-destination channel gain using NAF relaying is

$$
d(r)=n(1-r)+(1-2 r)^{+} .
$$

This result indicates that at multiplexing gains $r>0.5$ the relay does not play any role; the only available diversity at $r>0.5$ is that of multiuser diversity generated by selection among $n$ sources.

To prove the result, we make use of the calculation method in [18], [28]. An outline of the proof is as follows. We assume that $v_{1}$ is the exponential order of the random variable $\frac{1}{\left|h_{i^{*} d}\right|^{2}}$, i.e.,

$$
v_{1}=-\frac{\log \left(\left|h_{i^{*} d}\right|^{2}\right)}{\log \rho} .
$$

The probability density function of the exponential order is

$$
p_{v}=n \ln (\rho) \rho^{-v} e^{-\rho^{-v}}\left(1-e^{-\rho^{-v}}\right)^{n-1}
$$

which, asymptotically,

$$
p_{v} \doteq \begin{cases}0, & v<0 \\ \rho^{-n v}, & v \geq 0 .\end{cases}
$$

The probability of outage can be characterized by $P_{O} \doteq \rho^{-d_{o}}$ where

$$
d_{o}=\inf _{\left(v_{1}, v_{2}, u\right) \in O^{+}} n v_{1}+v_{2}+u
$$

where $v_{2}$ and $u$ are the exponential order of $1 /\left|h_{i^{*} r}\right|^{2}$ and $1 /\left|h_{r d}\right|^{2}$, respectively. The set $O$ characterizes the outage event and $O^{+}$is $O \cap R^{3+}$. Optimization problems of this form have been solved in [28] and also in the context of opportunistic relay networks we have demonstrated a solution in Appendix IV for the shared relay channel; therefore, we omit a similar solution here in the interest of brevity.

4) Dynamic Decode and Forward: The DMT of the opportunistic DDF MARC, where the selection is based on the source-destination channel gain, is given by

$$
d(r)= \begin{cases}(n+1)(1-r), & \frac{n}{n+1} \geq r \geq 0 \\ n \frac{(1-r)}{r}, & 1 \geq r \geq \frac{n}{n+1}\end{cases}
$$

The proof, which is omitted for brevity, follows [18], [28] together with Lemmas 1, 2, and 3 of this paper and the NAF MARC proof. The DDF achieves the optimal trade-off (the genie-aided DMT) for $\frac{n}{n+1} \geq r \geq 0$. For multiplexing gains $r>\frac{n}{n+1}$ the relay does not have enough time to perfectly help the selected source. However, as $n$ grows, the DMT approaches the upper bound (genie-aided).

5) Compress and Forward: The node selected by the opportunistic algorithm has index $i^{*}$. The system will be in outage if the transmission rate $r \log \rho$ is less than the instantaneous mutual information $I\left(x_{i^{*}} ; \hat{y}_{r}, y_{d} \mid x_{r}\right)$, where $\hat{y}_{r}$ represents the compressed signal at the relay, $y_{r}$ and $y_{d}$ are the received signals at the relay and the destination, respectively, and $x_{i *}$ and $x_{r}$ are the source and relay transmitted signals, respectively. Using selection scheme based on the direct link only and applying the same techniques as in [30], it follows that the $\mathrm{CF}$ protocol achieves the following DMT:

$$
d(r)=\min \left(d_{B C}(r), d_{M A C}(r)\right)
$$

where $d_{B C}, d_{M A C}$ correspond to the outage of broadcast and MAC cutsets, as follows:

$$
\begin{aligned}
d_{B C}(r) & \triangleq-\lim _{\rho \rightarrow \infty} \frac{\min _{p\left(x_{\left.i^{*}, x_{r}\right)}\right.} \mathbb{P}\left(I\left(x_{i^{*}} ; y_{r} y_{d} \mid x_{r}\right)<r \log \rho\right)}{\log \rho} \\
& =-\lim _{\rho \rightarrow \infty} \frac{\mathbb{P}\left(\log \left|I+\rho H_{B C} H_{B C}^{\dagger}\right|<r \log \rho\right)}{\log \rho} \\
d_{M A C}(r) & \triangleq-\lim _{\rho \rightarrow \infty} \frac{\min _{p\left(x_{\left.i^{*}, x_{r}\right)}\right.} \mathbb{P}\left(I\left(x_{i^{*}} x_{r} ; y_{d}\right)<r \log \rho\right)}{\log \rho} \\
& =-\lim _{\rho \rightarrow \infty} \frac{\mathbb{P}\left(\log \left|I+2 \rho H_{M A C} H_{M A C}^{\dagger}\right|<r \log \rho\right)}{\log \rho} .
\end{aligned}
$$

The transmit signals $x_{i^{*}}$ and $x_{r}$ are from random codebooks that are drawn according to complex Gaussian dis- 
tributions with zero mean and variance $\sqrt{\rho}$. We define $H_{B C} \triangleq\left[\begin{array}{c}h_{i^{*} r} \\ h_{i^{*}}\end{array}\right], H_{M A C} \triangleq\left[h_{i^{*} d} h_{r d}\right]$ and ()$^{\dagger}$ denotes the Hermitian operator. The derivation of (49), (50) uses the fact that a constant scaling in the transmit power does not change the DMT [18].

Using the techniques in [18], [28] and following the NAF MARC DMT proof, it is possible to calculate the following:

$$
\begin{aligned}
d_{B C}(r)= \begin{cases}(n+1)-\frac{r}{t}, & r \leq t<\frac{1}{n+1} \\
n \frac{(1-r)}{(1-t)}, & t<\min \left(r, \frac{1}{n+1}\right) \\
(n+1)(1-r), & t \geq \frac{1}{n+1}\end{cases} \\
d_{M A C}(r)= \begin{cases}(n+1)-\frac{r}{1-t}, & 1-r \geq t>\frac{n}{n+1} \\
n \frac{(1-r)}{t}, & t>\max \left\{1-r, \frac{n}{n+1}\right\} \\
(n+1)(1-r), & t \leq \frac{n}{n+1}\end{cases}
\end{aligned}
$$

Details of the derivation are similar to, e.g., Theorem 4 and are omitted for brevity.

From (48)-(52), it follows that the genie aided DMT upper bound can be achieved for any value of $\frac{1}{n+1} \leq t \leq \frac{n}{n+1}$. The maximum achieved DMT is given by

$$
d(r)=(n+1)(1-r)^{+} .
$$

\section{Optimality of the Achievable DMTs}

Although the previous DMTs were calculated using simplified selection schemes that only observed the source-destination direct link, one can show that for each of the relaying protocols, no improvement in DMT is possible by more sophisticated selection schemes.

This fact is self-evident for the $\mathrm{CF}$ relaying result, since it meets the genie-aided upper bound. The NAF and DDF do not meet the genie-aided bound; therefore, it is not obvious that they perform optimally under the simplified selection scheme. We now proceed to investigate this question for DDF and NAF.

The DMT of the multiple access relay channel with opportunistic user selection is given by

$$
d(r)=\lim _{\rho \rightarrow \infty} \frac{\log \mathbb{P}\left(\mathcal{O}_{1}, \ldots, \mathcal{O}_{n}\right)}{\log \rho}
$$

where $\mathcal{O}_{i}$ represents the outage event for the access mode characterized by source $i$ transmitting to the destination with the help of the relay.

In a manner similar to [28] and (129), the probability of outage $\mathbb{P}\left(\mathcal{O}_{1}, \ldots, \mathcal{O}_{n}\right)$ can be expressed as follows:

$$
\mathbb{P}\left(\mathcal{O}_{1}, \ldots, \mathcal{O}_{n}\right) \doteq \rho^{-d_{o}(r)}
$$

where

$d_{o}(r)=\inf _{\left(v_{1}^{(1)}, u^{(1)}, \ldots, v_{1}^{(n)}, u^{(n)}, v_{2}\right) \in O} v_{2}+\sum_{j=1}^{n}\left(v_{1}^{(j)}+u^{(j)}\right)$

The random variables $v_{1}^{(j)}, u^{(j)}$ and $v_{2}$ represent the exponential order of $1 /\left|h_{j d}\right|^{2}, 1 /\left|h_{j r}\right|^{2}$ and $1 /\left|h_{r d}\right|^{2}$, respectively. Each of these random variables has a probability density function that is asymptotically equal to

$$
p(x) \doteq \begin{cases}0, & x<0 \\ \rho^{-x}, & x \geq 0 .\end{cases}
$$

The set $O$ represents the outage event for the opportunistic network. We know $O=\mathcal{O}_{1}^{+} \cap \ldots \cap \mathcal{O}_{n}^{+}$, i.e., the opportunistic system is considered in outage when no access mode is viable.

For NAF the outage region is defined by [28]

$$
\begin{aligned}
\mathcal{O}_{j}^{+} & =\left\{\left(v_{1}^{(j)}, v_{2}, u^{(j)}\right) \in R^{3+} \mid(l-2 m)\left(1-v_{1}^{(j)}\right)^{+}\right. \\
& \left.\left.+m \max \left\{2\left(1-v_{1}^{(j)}\right), 1-\left(v_{2}+u^{(j)}\right)\right\}\right)^{+}<r l\right\}
\end{aligned}
$$

where $\mathrm{m}$ is rank of the relay amplification matrix and $l$ is the block length. The solution to (55) and (57) is facilitated by the knowledge that $d_{o}(r)$ is maximized when $m=l / 2$, leading to

$$
d_{N A F}(r)=n(1-r)+(1-2 r) .
$$

This is the best diversity obtained for NAF, which is similar to the simplified selection based on the source-destination link. Therefore, the optimality of the simplified selection rule is established for NAF.

For DDF the outage region is defined by [28]

$$
\begin{aligned}
\mathcal{O}_{j}^{+}=\{( & \left.v_{1}^{(j)}, v_{2}, u^{(j)}\right) \in R^{3+} \mid t^{(j)}\left(1-v_{1}^{(j)}\right)^{+} \\
& \left.+\left(1-t^{(j)}\right)\left(1-\min \left(v_{1}^{(j)}, v_{2}\right)\right)^{+}<r\right\}
\end{aligned}
$$

where $t^{(j)}$ is the listening-time ratio of the half-duplex relay when source $j$ is transmitting, with $r \leq t^{(j)} \leq 1$. In the following we outline the solution of (55) and (59) for a two-user MARC. The generalization to $n$ users is straight forward.

Our strategy for solving the optimization problem is to partition the optimization space into eight regions, solve the optimization problem over each region as a function of $t^{(1)}$ and $t^{(2)}$, maximize over $t^{(1)}$ and $t^{(2)}$ and then find the minimum of the eight solutions. The eight regions correspond to the Cartesian product of whether each of the three positive variables $v_{1}^{(1)}, v_{1}^{(2)}, v_{2}$ is greater than or less than 1 . Following the calculations, which are straight forward, the DMT for DDF is

$$
d_{D D F}(r)= \begin{cases}(n+1)(1-r) & \frac{n}{n+1} \geq r \geq 0 \\ n \frac{1-r}{r} & 1 \geq r>\frac{n}{n+1}\end{cases}
$$

which matches the DMT of simplified selection based on the source-destination links. Therefore, the optimality of simplified selection for the DDF is established.

We can follow essentially the same steps for the broadcast relay channel and obtain the same DMTs for both the NAF and DDF. The optimization problem in the broadcast case is slightly different: the shared link in BRC is the source-relay channel while it is the relay-destination channel in the MARC. Nevertheless, very similar strategies follow through for the BRC with only small adjustments. 


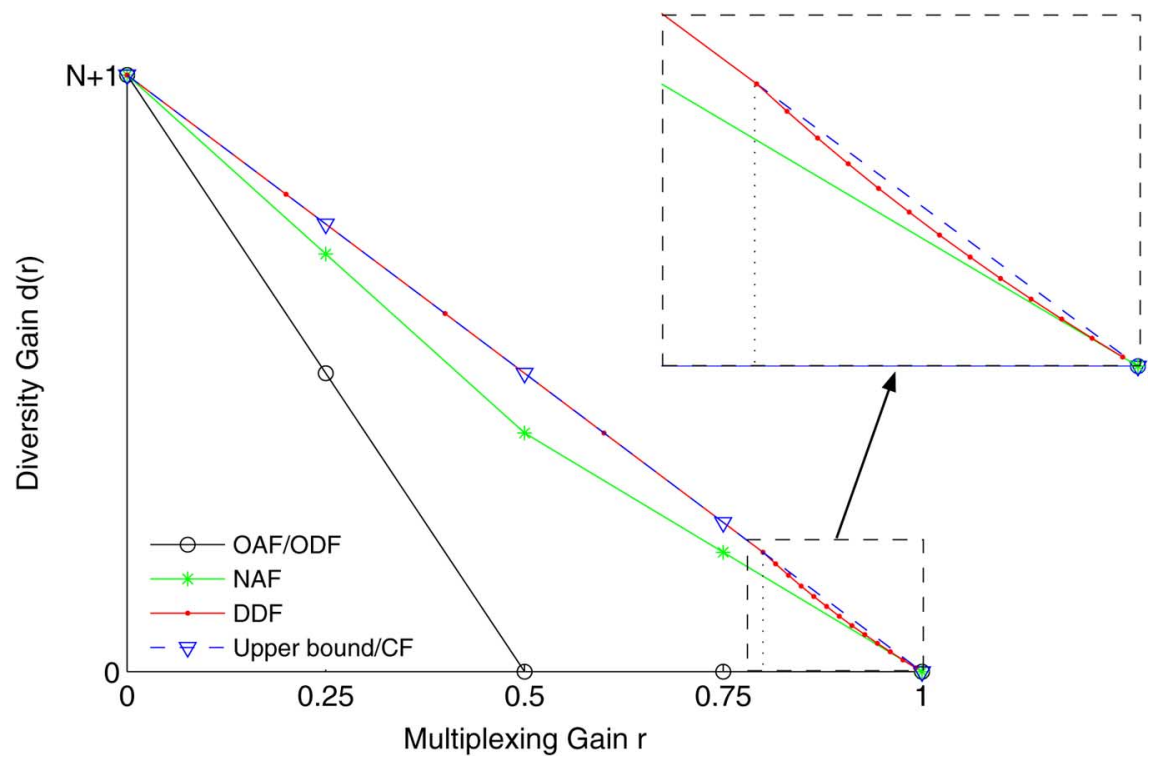

Fig. 12. DMT for a N-user opportunistic multiple-access relay channel. The insert shows the high-multiplexing gain region.

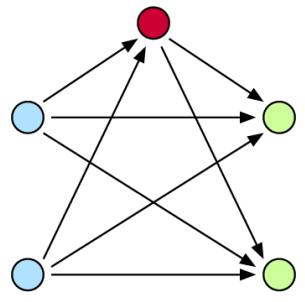

Fig. 13. X-relay channel.

\section{Opportunistic X-Relay ChanNel}

The X-relay channel is defined as a $n \times n$ node wireless network with a relay, where each of the $n$ sources has messages for each of the $n$ destinations (see Fig. 13). The sources are not allowed to cooperate with each other, but the relay cooperates with all sources.

There are only a few results available on the $\mathrm{X}$ channel, among them, it has been shown [43] that the $2 \times 2 \mathrm{X}$-channel with no relay has exactly $\frac{4}{3}$ degrees of freedom when the channels vary with time and frequency. The X-relay channel introduces a relay to the $\mathrm{X}$ channel for improved performance. For the purposes of exposition we focus on a $2 \times 2 \mathrm{X}$-relay channel, but the results are extendable to the $n \times n$ channel.

The opportunistic X-relay channel has four access modes as shown in Fig. 14. These modes avoid interference across different message streams and satisfy our working definition of opportunistic modes in relay networks.

\section{A. DMT Upper Bound}

To find an upper bound for the DMT of opportunistic X-relay channel, we assume a genie transfers the data from the sources to the relay and also allows the sources to know each other's messages. For the upper bound we also allow the destinations to fully cooperate, noting that it can only improve the performance. Fig. 15 shows the genie-aided opportunistic modes, where the two-sided arrows indicate the free exchange of information by

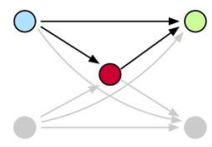

(a)

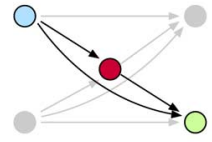

(c)

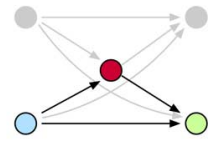

(b)

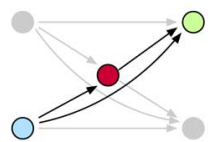

(d)
Fig. 14. Opportunistic modes of the X-relay channel.

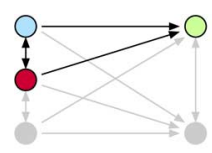

(a)

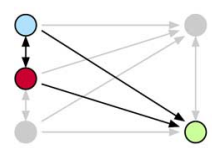

(c)

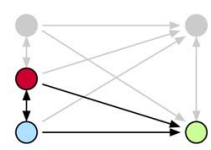

(b)

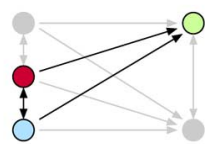

(d)
Fig. 15. Opportunistic modes of genie-aided X-relay channel.

the genie. From this figure, it is easy to see that the genie-aided $\mathrm{X}$-relay channel is equivalent to a MIMO system with 3 transmit antennas and 2 receive antennas.

The performance of the opportunistic X-relay channel is, therefore, upper bounded by a $3 \times 2$ MIMO system with antenna selection, choosing for each codeword two transmitting and one receiving antennas. It is noteworthy that the $3 \times 2$ antenna selection allows one configuration that does not have a counterpart in the opportunistic modes in the X-relay channel; therefore, due to the extra flexibility, the MIMO system with antenna selection upper bounds the performance of the genie-aided opportunistic X-relay channel. 


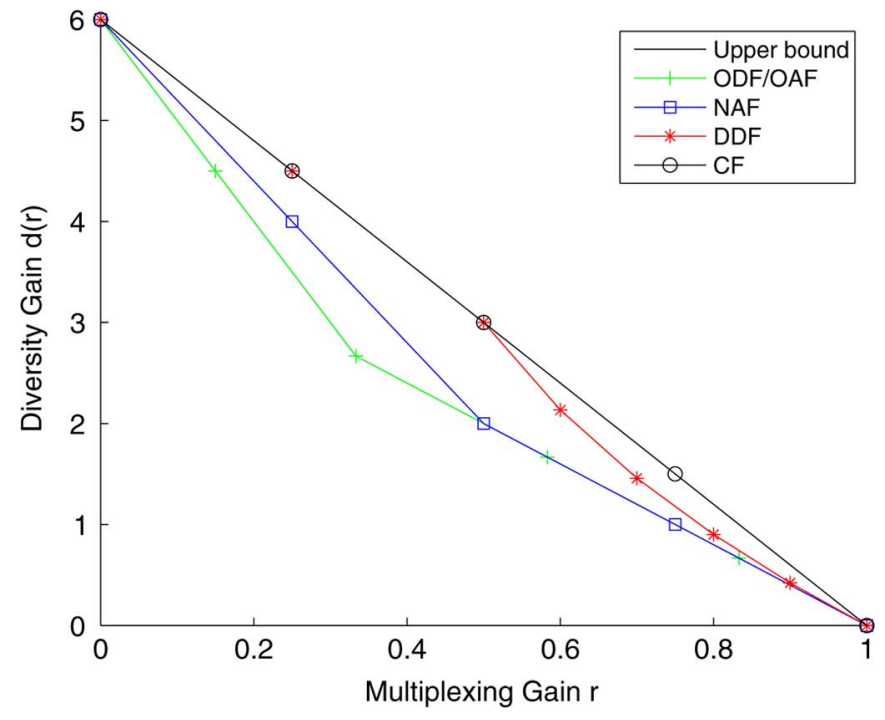

Fig. 16. DMT of the opportunistic X-relay channel under orthogonal AF and DF, Nonorthogonal AF, Dynamic DF, and CF. The CF achieves the DMT upper bound.

Using the result from (29), a $3 \times 2$ MIMO system with two antennas selected from the transmitter side and one antenna selected from the receiver side has a DMT that is upper bounded by $d(r)=6(1-r)^{+}$. This in turn is an upper bound to the performance of the opportunistic X-relay channel.

\section{B. Achievable DMT}

For deriving achievable rates, we consider the following simplified opportunistic scheme. First, we choose between the two access modes (a) and (b) in Fig. 14. If both these two modes are in outage, we consider only the direct link of the two access modes (c) and (d), i.e., the relay is not allowed to cooperate in modes (c) and (d). Note that this is only a simplification for the purposes of achievable-DMT analysis, the idea being that if the relay is useful in neither of the access modes (a) and (b), it is unlikely to be useful at all. The approximation involving the conditional removal of the relay from (c) and (d) allows the access modes to become independent and simplifies the analysis. The resulting achievable rate is tight against the upper bound for compress-forward, as seen in the sequel, but not demonstrably so for other protocols.

Access modes (a) and (b) do not share any common links; therefore, their statistics are independent. Each of them is an ordinary relay channel which can achieve $d(r)=2(1-r)^{+}$ via the CF protocol [30]. The (c) and (d) access modes, which were reduced to a single link, each achieves the DMT $d(r)=$ $(1-r)^{+}$. Furthermore, the source-destination links in (c) and (d) are disjoint from the links in (a) and (b); therefore, the statistics are independent, and we can use Lemma 1 to find the overall DMT $d(r)=6(1-r)^{+}$. Note that this achievable DMT meets the upper bound; therefore, the DMT of the X-relay channel under CF is exactly $d(r)=6(1-r)^{+}$.

Achievability results for relaying protocols other than CF can be obtained along the same lines. We begin with NAF. Recall that the DMT of a simple relay network (source, relay, destination) under NAF is $d(r)=(1-r)^{+}+(1-2 r)^{+}$. Combining the four access modes (a), (b), (c), (d) mentioned earlier for the $\mathrm{X}$-relay channel together with the NAF protocol results in

$$
\begin{aligned}
d_{X N A F}(r) & =2(1-r)^{+}+2\left[(1-r)^{+}+(1-2 r)^{+}\right] \\
& =4(1-r)^{+}+2(1-2 r)^{+}
\end{aligned}
$$

A similar result exists for the DDF where the DMT is given by

$$
d_{X D D F}(r)= \begin{cases}6(1-r), & 0 \leq r<\frac{1}{2} \\ 2 \frac{1-r}{r}+2(1-r), & \frac{1}{2} \leq r \leq 1 .\end{cases}
$$

Applying the same analysis to orthogonal AF and DF yields a diversity $d(r)=2(1-r)^{+}+4(1-2 r)^{+}$, but there is more to be said for orthogonal transmission. In orthogonal transmission it may be beneficial at high multiplexing gains to shut down the relay; therefore, a complete analysis requires two more opportunistic modes that are derived by shutting down the relay from modes (a) and (b). Using this extended set of six access modes, the DMT of the opportunistic X-relay channel with orthogonal $\mathrm{AF}$ or orthogonal DF is

$$
d(r)=4(1-r)^{+}+2(1-2 r)^{+}
$$

which matches the DMT of NAF.

Thus far, to find achievable DMTs for the X relay channel we used simplified selection rules and access modes. In the case of CF, this simplified achievable DMT is in fact optimal since it matches the genie upper bound. One can show that for NAF and DDF also, no DMT gains can be obtained by more sophisticated selection rules and access modes, as outlined below.

To find the overall optimal DMT without the simplifications, we need to solve a linear optimization problem similar to (55) where

$$
\begin{aligned}
& d_{o}(r)= \\
& \inf _{\left(v_{1}^{(i j)}, v_{2}^{(r j)}, u^{(j r)}\right) \in O, i, j \in\{1,2\}} \sum_{j=1}^{2}\left(\sum_{i=1}^{2} v_{1}^{(i j)}+v_{2}^{(r j)}+u^{(j r)}\right)
\end{aligned}
$$

where $v_{1}^{(i j)}, v_{2}^{(r j)}$ and $u^{(j r)}$ represent the exponential order of $1 /\left|h_{i j}\right|^{2}, 1 /\left|h_{r j}\right|^{2}$ and $1 /\left|h_{j r}\right|^{2}$, respectively. The outage event $O$ is characterized by $\mathcal{O}_{1}^{+} \cap \mathcal{O}_{2}^{+} \cap \mathcal{O}_{3}^{+} \cap \mathcal{O}_{4}^{+}$, i.e., the system is in outage if all access modes are in outage. The outage event is given by (57) for NAF and (59) for DDF. In a straight forward manner, the optimization above gives the same DMTs found by the simplified selection criterion; therefore, the calculated DMTs cannot be improved upon and are optimal.

\section{Gateway Channel}

The gateway channel [44] is a multinode network with $M$ source-destination pairs that communicate with the help of a relay (see Fig. 17). Each source communicates only with its corresponding destination. A two-hop communication scheme is used, where at the first hop the sources transmit to the relay and at the second hop the relay transmits to the destinations. No direct link exists between the sources and destinations; therefore, if the relay is in outage, the destination will surely be in outage. Under these conditions, the most natural mode of operation is decode-and-forward, although amplify-and-forward may also be considered due to practical limitations. In this work we concentrate on the DF gateway channel. 


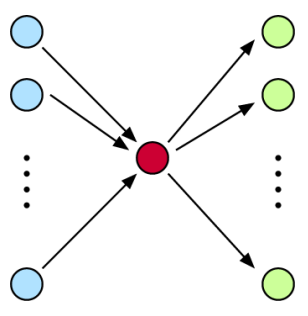

Fig. 17. Gateway channel.

With an infinite buffer at the relay, the gateway channel decomposes into a concatenation of a MAC and a broadcast channel. An infinite buffer would thus simplify the analysis but also increase the overall latency and relay complexity. One of the interesting outcomes of the forthcoming analysis is that that data buffering in the asymptotic high-SNR regime does not provide a performance advantage (in the sense of DMT).

We start with the nonopportunistic gateway channel, and then move to the opportunistic scheme.

\section{A. No Transmit CSI}

We first consider the case where all nodes have receive-side CSI, but the nodes, and in particular the relay, do not have transmit-side CSI. Under these conditions, we cannot choose source-destination pairs according to their SNR. Then the choice of transmission strategies on the MAC and broadcast side of the network are as follows.

On the broadcast side, the channel gains are random and unknown to the relay. In light of symmetric rate requirements, the transmit strategy must be symmetric with respect to the destinations. Under this symmetry, the best achievable rate is according to orthogonal transmission [45] and superposition coding does not give better results.

For the multiple-access side, under symmetric rate requirement, both orthogonal and superposition channel access are viable. It has been shown that superposition access gives slightly better performance at medium SNR, while at high and low SNR the two methods have asymptotically the same capacity under symmetric rates [45, pp. 243-245].

In the absence of transmit-side CSI, and with symmetric rate requirements, the network does indeed decompose into a cascade of a multiple-access and a broadcast subnetworks, and the overall outage probability is given by

$$
\begin{aligned}
P_{\mathcal{O}} & =1-\left(1-P_{M A C}\right)\left(1-P_{B C}\right) \\
& =P_{M A C}+P_{B C}-P_{M A C} P_{B C} .
\end{aligned}
$$

Where $P_{M A C}$ (respectively $P_{B C}$ ) denotes the outage of the MAC (respectively broadcast channel), defined as the probability that one or more of the users in the MAC (respectively broadcast channel) cannot support rate $R$. In a slowly fading environment, for a power allocation vector $P_{s}=\left(P_{1}, \ldots, P_{M}\right)$, a fading state $H=\left(h_{1 r}, \ldots, h_{M r}\right)$ and superposition coding, the outage is given by $P_{M A C}=\mathbb{P}\left(\bar{R} \notin \mathcal{C}_{M A C}\right)$ where

$\mathcal{C}_{M A C}(H, P)=\left\{\bar{R}: \sum_{i \in S} R_{i} \leq \frac{1}{2} \log \left(1+\frac{1}{N} \sum_{i \in S}\left|h_{i r}\right|^{2} P_{i}\right)\right\}$.

With a time-sharing MAC, the outage probability is

$$
P_{M A C}=\mathbb{P}\left(\left\{R_{i}>\frac{1}{2 M} \log \left(1+\rho\left|h_{i r}\right|^{2}\right), i=1, \ldots, M\right\}\right) .
$$

On the broadcast side, the outage is given by $P_{B C}=\mathbb{P}(\bar{R} \notin$ $\left.\mathcal{C}_{B C}\right)$, where

$$
\mathcal{C}_{B C}=\left\{\bar{R}: R_{i} \leq \frac{1}{2 M} \log \left(1+\rho\left|h_{r i}\right|^{2}\right)\right\} .
$$

Without transmit CSI, the DMT is the minimum of the DMT of the MAC and the broadcast channel. For the MAC channel, it has been shown [19] that for multiplexing gains $r \leq \frac{M}{M+1}$, the diversity $d=1-r / M$ is achievable, while for higher rates $\frac{M}{M+1}<r \leq 1$, the diversity of $d=M(1-r)$ is obtained.

For the broadcast channel, since time sharing achieves the maximum sum-rate bound, the broadcast DMT is similar to the single-user DMT. The DMT of the network is bounded by the DMT of the broadcast part of the network. Thus, including the half-duplex consideration, the best achievable DMT is

$$
d(r)=(1-2 r)^{+} .
$$

The same DMT can be obtained with orthogonal channel access; superposition coding has no effect on the DMT.

\section{B. Opportunistic Channel Access}

In this scenario, the relay is assumed to have channel state information (either perfect or incomplete) about its incoming and outgoing links. Using this information, during each transmission interval the relay selects the best overall source-destination pair and gives it access to the channel. Form Lemma 1, it is easy to see that the DMT of an opportunistic gateway channel is upper bounded by $d(r) \leq n(1-2 r)^{+}$. We start by assuming perfect CSI at the relay.

1) Full CSI at the Relay: We start by defining

$$
\gamma_{i} \triangleq \min \left(\left|h_{i r}\right|^{2},\left|h_{r i}\right|^{2}\right) .
$$

In the decode-and-forward protocol, end-to-end data transmission is feasible if and only if both source-relay and relay-destination links can support the desired rate; therefore, $\gamma_{i}$ is the effective channel gain that governs the rate supported by a DF protocol for any node pair $i$. In the opportunistic mode, we would like to support the maximum instantaneous rate; therefore, user $i^{*}$ will be selected such that

$$
i^{*}=\arg \max _{i} \gamma_{i}
$$


We now investigate the statistics of $\gamma_{i^{*}}$. Since the channel fading coefficients $h_{r i}$ and $h_{i r}$ are complex Gaussian random variables, the channel gains $\left|h_{r i}\right|^{2}$ and $\left|h_{i r}\right|^{2}$ obey exponential distributions with exponential parameters $\frac{1}{E\left[\left|h_{r_{i}}\right|^{2}\right]}$ and $\frac{1}{E\left[\left|h_{i r}\right|^{2}\right]}$, respectively. It is known that the minimum of $M$ exponential random variables with parameters $\lambda_{k}$ is an exponential random variable with parameter $\sum_{k=1}^{M} \lambda_{k}$; therefore, the pdf of $\gamma_{i}$ is an exponential distribution with parameter $\lambda=2$. Therefore, the cdf of the maximum SNR for all the source-relay-destinations links $\gamma_{i^{*}}$ is

$$
F_{\gamma_{i^{*}}}(x)=\left(1-e^{-2 x}\right)^{M} .
$$

The network is considered in outage when none of the sourcedestination pairs can support the desired transmission rate $R$. The outage condition is therefore

$$
\begin{aligned}
P_{\mathcal{O}} & =\mathbb{P}\left(R>\frac{1}{2} \log \left(1+\rho \gamma_{i^{*}}\right)\right) \\
& =\mathbb{P}\left(\gamma_{i^{*}}<\frac{\rho^{2 r}-1}{\rho}\right) \\
& =\left(1-\exp \left(-2 \frac{\rho^{2 r}-1}{\rho}\right)\right)^{M} .
\end{aligned}
$$

The block sizes in our analyses are large enough so that the error events are dominated by outage events; therefore, the probability of error can be approximated by the outage probability. Using the Taylor approximation $1-\exp (-x) \approx x$, we get:

$$
\begin{aligned}
P_{e} & \doteq\left(\frac{\rho^{2 r}-1}{\rho}\right)^{M} \\
& \doteq \rho^{-M(1-2 r)} .
\end{aligned}
$$

where the Taylor approximation is valid for $2 r<1$. Hence, the opportunistic gateway channel achieves the following DMT:

$$
d(r)=M(1-2 r)^{+}
$$

Remark 6: If the path selection criterion uses one set of channel gains, i.e., either $\left\{h_{i r}\right\}$ alone or $\left\{h_{r i}\right\}$ alone, no diversity gain would result. For example, selecting on the MAC side of the network would give $\gamma_{i}=\min \left(\left|h_{i^{*} r}\right|^{2},\left|h_{r i^{*}}\right|^{2}\right)$ where $i^{*}=\arg \max \left|h_{i r}\right|^{2}$. Since the channel gains on the two sides are independent, $\left|h_{r i^{*}}\right|^{2}$ is still exponential and dominates the diversity order.

Remark 7: The outage calculations assume that upon selection each source must be connected to its corresponding destination within one transmission interval, implying that no long-term storage and buffering is taking place at the relay. In addition to simplifying the relay, this is also helpful in terms of reducing the end-to-end delay due to opportunistic communication.

Remark 8: An infinite buffer at the relay may increase the throughput, but it does not improve the DMT. If the relay can hold onto the data, the incoming packets could wait indefinitely until the path to their destination is dominant. Under this condition, the opportunistic MAC and opportunistic broadcast operations can be performed independently, each giving rise to a diversity $d=M(1-2 r)^{+}$; thus, the overall diversity would also be $d=M(1-2 r)^{+}$. However, this is no more than the diversity obtained without the buffer.

To summarize, a buffer would not improve the DMT, however, it would allow us to achieve the optimal DMT via local decision making (using MAC information on the MAC side, and broadcast channel information on the broadcast side). Without buffering, the relay must make decisions jointly in order to achieve optimal DMT.

2) Limited Feedback: We now assume the relay does not have perfect CSI but rather has access to one bit of information per node from each destination and is further able to send one bit of information per node to each of the sources. We wish to explore the DMT of this network under the one-bit feedback strategy.

Each destination node knows its incoming channel gain via the usual channel estimation techniques. Each destination compares its incoming channel gain to a threshold $\alpha$, reporting the result via the one-bit feedback to the relay. The $k$ destination nodes that report " 1 "(and their respective channels) are characterized as eligible for data transmission in that interval. From among these $k$ eligible destinations, the relay chooses the one whose corresponding source-relay channel is the best.

The network is considered in outage if there is no sourcerelay-destination link that can support the target rate $R$. We design the threshold of the second hop of the network such that each destination reports " 1 " if the corresponding relay-destination link can support this rate $R$, i.e., $\alpha=\frac{\rho^{2 r}-1}{\rho}$. The outage event occurs if no destination reports positively, or if some destinations are eligible, but none of the corresponding source-relay links can support the rate $R$. If according to this methodology the relay detects more than one end-to-end path that can support the rate $R$, the relay selects one of them randomly.

We define $A_{m}$ as the event of $m$ destinations reporting "1", and $\mathbb{P}\left(e \mid A_{m}\right)$ as the probability of error given that $m$ destinations report " 1 ". This is the probability that none of the $m$ eligible relay-destination channels have a corresponding sourcerelay link that can support the rate $R$. The probability of outage in this case is

$$
P_{\mathcal{O}}=\mathbb{P}\left(A_{0}\right)+\sum_{m=1}^{M} \mathbb{P}\left(A_{m}\right) \mathbb{P}\left(e \mid A_{m}\right) .
$$

The probability of $m$ destinations reporting " 1 " and $M-i$ destinations reporting " 0 " is

$$
\begin{aligned}
\mathbb{P}\left(A_{m}\right) & =\left(\begin{array}{c}
M \\
m
\end{array}\right) F_{\gamma}(\alpha)^{m}\left(1-F_{\gamma}(\alpha)\right)^{M-m} \\
& =\left(\begin{array}{c}
M \\
m
\end{array}\right)\left(e^{-\lambda \alpha}\right)^{m}\left(1-e^{-\lambda \alpha}\right)^{M-m}
\end{aligned}
$$

where $F_{\gamma}(x)$ is the cdf of the channel gains $\gamma=|h|^{2}$, which is exponentially distributed with parameter $\lambda=1$. The probability of error given that $m$ destinations report " 1 " is

$$
\begin{aligned}
\mathbb{P}\left(e \mid A_{m}\right) & =\mathbb{P}\left(\max _{j \in S}\left|h_{j r}\right|^{2} \leq \alpha\right) \\
& =\left(1-F_{\gamma}(\alpha)\right)^{m}=\left(1-e^{-\lambda \alpha}\right)^{m}
\end{aligned}
$$


where $S \subset\{1, \ldots, M\},|S|=m$, and we use the fact that source-relay and relay-destination channel gains have the same distribution $F_{\gamma}$. Assuming nonidentical exponential distributions introduces more variables into analysis but the end results will be identical. Substituting (76), (77) in (75), the outage probability becomes

$$
\begin{aligned}
P_{\mathcal{O}} & =\left(1-e^{-\lambda \alpha}\right)^{M} \\
& +\sum_{m=1}^{M}\left(\begin{array}{c}
M \\
m
\end{array}\right)\left(e^{-\lambda \alpha}\right)^{m}\left(1-e^{-\lambda \alpha}\right)^{M-m}\left(1-e^{-\lambda \alpha}\right)^{m} \\
& =\sum_{m=0}^{M}\left(\begin{array}{c}
M \\
m
\end{array}\right)\left(e^{-\lambda_{g} \alpha}\right)^{m}\left(1-e^{-\lambda \alpha}\right)^{M-m}\left(1-e^{-\lambda \alpha}\right)^{m} .
\end{aligned}
$$

To calculate the DMT, from (75), the outage probability is

$$
\begin{aligned}
P_{\mathcal{O}}= & \mathbb{P}\left(\frac{1}{2} \log \left(1+\max _{i}\left|h_{r i}\right|^{2} \rho\right) \leq r \log \rho\right) \\
+ & \sum_{m=1}^{M}\left(\begin{array}{c}
M \\
m
\end{array}\right) \mathbb{P}\left(\frac{1}{2} \log \left(1+\left|h_{r d}\right|^{2}\right) \leq r \log \rho\right)^{M-m} \\
& \quad \times \mathbb{P}\left(\frac{1}{2} \log \left(1+\left|h_{r d}\right|^{2}\right) \geq r \log \rho\right)^{m} \\
& \quad \times \mathbb{P}\left(\frac{1}{2} \log \left(1+\max _{j \in S,|S|=m}\left|h_{j r}\right|^{2}\right) \leq r \log \rho\right) \\
\doteq & \mathbb{P}\left(\max _{i}\left|h_{r i}\right|^{2} \leq \rho^{2 r-1}\right) \\
+ & \sum_{m=1}^{M} \mathbb{P}\left(\left|h_{r d}\right|^{2} \leq \rho^{2 r-1}\right)^{M-m} \mathbb{P}\left(\left|h_{r d}\right|^{2} \geq \rho^{2 r-1}\right)^{m} \\
\doteq & \times \mathbb{P}\left(\max _{j \in S,|S|=m}^{M}\left|h_{j r}\right|^{2} \leq \rho^{2 r-1}\right) \\
\doteq & \sum_{m=1}^{M(2 r-1)} .
\end{aligned}
$$

So, we have

$$
d(r)=M(1-2 r)^{+} .
$$

Thus, even 1-bit feedback is enough to achieve optimal DMT.

\section{Conclusion}

The high-SNR performance of opportunistic relay networks are investigated. Except for a handful of simple relay selection scenarios, there are two main difficulties in the analysis of opportunistic relay networks: (1) the decision variables often depend on more than one link gain, complicating the performance analysis and (2) the opportunistic modes may share links and thus are statistically dependent, which complicates the order statistics that govern the performance of opportunistic systems. In this work, several relaying geometries are studied and the corresponding DMTs are developed for a number of well-known relaying protocols, including the $\mathrm{AF}, \mathrm{DF}, \mathrm{CF}, \mathrm{NAF}$, and DDF. In several instances, selection schemes based on the direct source- destination links are shown to achieve optimal performance, for example the CF multiple access channel. In some network geometries, opportunistic selection using 1-bit feedback is shown to achieve the optimal DMT performance.

\section{APPENDIX I \\ OPPORTUNISTIC DF ORTHOGONAL RELAYING Over a Simple Relay CHANNEl}

The DMT of the opportunistic orthogonal relaying is given by

$$
d(r)=d_{1}(r)+d_{2}(r)
$$

where

$$
\begin{aligned}
& d_{1}(r)=\lim _{\rho \rightarrow \infty} \frac{\log \mathbb{P}\left(e_{1}\right)}{\log \rho} \\
& d_{2}(r)=\lim _{\rho \rightarrow \infty} \frac{\log \mathbb{P}\left(e_{2} \mid e_{1}\right)}{\log \rho} .
\end{aligned}
$$

The events $e_{1}$ and $e_{2}$ represent the error in the nonrelay and the relay-assisted modes, respectively. The nonrelay access mode is a simple direct link, whose DMT is $d_{1}(r)=(1-r)^{+}$. The DMT of the relay-assisted access mode is known; however, the DMT of the relay channel conditioned on the outage event of the direct link requires new calculations.

Recall that the orthogonal DF relaying works as follows: The transmission interval is divided into two halves. In the first half, the source transmits. If the relay cannot decode the source message, it will remain silent and the source will continue to transmit into the second half-interval. If the relay decodes the source message, the relay forwards the decoded message to the destination in the second half of the transmission interval and the source remains silent.

Because of orthogonality and with the use of long codewords, it is trivial to see that error is dominated by outage. The conditional outage probability of the relay-assisted mode is given by

$$
\begin{aligned}
\mathbb{P}\left(\mathcal{O}_{2} \mid \mathcal{O}_{1}\right)=\mathbb{P} & \left(\left\{\frac{1}{2} \log (1+U \rho)<r \log \rho\right\} \mid\right. \\
& \left.\left\{\log \left(1+\left|h_{s d}\right|^{2} \rho\right)<r \log \rho\right\}\right) \\
= & \mathbb{P}\left(\left\{U<\frac{\rho^{2 r}-1}{\rho}\right\} \mid\left\{\left|h_{s d}\right|^{2}<\frac{\rho^{r}-1}{\rho}\right\}\right)
\end{aligned}
$$

where the random variable $U$ is given by

$$
U= \begin{cases}2\left|h_{s d}\right|^{2}, & \left|h_{s r}\right|^{2}<\frac{\rho^{2 r}-1}{\rho} \\ \left|h_{s d}\right|^{2}+\left|h_{r d}\right|^{2}, & \left|h_{s r}\right|^{2} \geq \frac{\rho^{2 r}-1}{\rho} .\end{cases}
$$

The cdf of $U$ is given by

$$
\begin{aligned}
F_{U}(u)= & \mathbb{P}\left(\left|h_{s d}\right|^{2}<\frac{u}{2}\right) \mathbb{P}\left(\left|h_{s r}\right|^{2}<\frac{\rho^{2 r}-1}{\rho}\right) \\
& +\mathbb{P}\left(\left|h_{s d}\right|^{2}+\left|h_{r d}\right|^{2}<u\right) \mathbb{P}\left(\left|h_{s r}\right|^{2} \geq \frac{\rho^{2 r}-1}{\rho}\right) .
\end{aligned}
$$


Hence

$$
\begin{aligned}
\mathbb{P}\left(\mathcal{O}_{2} \mid \mathcal{O}_{1}\right)= & \mathbb{P}\left(\left\{\left|h_{s d}\right|^{2}<\frac{1}{2} \frac{\rho^{2 r}-1}{\rho}\right\} \mid\left\{\left|h_{s d}\right|^{2}<\frac{\rho^{r}-1}{\rho}\right\}\right) \\
& \times \mathbb{P}\left(\left|h_{s r}\right|^{2}<\frac{\rho^{2 r}-1}{\rho}\right) \\
+\mathbb{P}\left(\left\{\left|h_{s d}\right|^{2}+\left|h_{r d}\right|^{2}<\frac{\rho^{2 r}-1}{\rho}\right\} \mid\left\{\left|h_{s d}\right|^{2}<\frac{\rho^{r}-1}{\rho}\right\}\right) & \\
& \times \mathbb{P}\left(\left|h_{s r}\right|^{2} \geq \frac{\rho^{2 r}-1}{\rho}\right)
\end{aligned}
$$

One can show that $\frac{1}{2} \frac{\rho^{2 r}-1}{\rho}>\frac{\rho^{r}-1}{\rho}$, therefore

$$
\mathbb{P}\left(\left\{\left|h_{s d}\right|^{2}<\frac{1}{2} \frac{\rho^{2 r}-1}{\rho}\right\} \mid\left\{\left|h_{s d}\right|^{2}<\frac{\rho^{r}-1}{\rho}\right\}\right) \doteq 1 \text {. }
$$

To analyze the second conditional term in (87), we begin with the pdf of $Z=\left|h_{s d}\right|^{2}+\left|h_{r d}\right|^{2}$ conditioned on the event $B=\left\{\left|h_{s d}\right|^{2}<\frac{\rho^{r}-1}{\rho}\right\}$. The channel gain $\gamma \triangleq\left|h_{s d}\right|^{2}$ has the following conditional distribution:

$$
f_{\gamma \mid B}(x)= \begin{cases}\frac{e^{-x}}{1-e^{-\frac{\rho^{r}-1}{\rho}},}, & x \leq \frac{\rho^{r}-1}{\rho} \\ 0, & x>\frac{\rho^{r}-1}{\rho} .\end{cases}
$$

Defining $g_{1}(r, \rho) \triangleq \frac{\rho^{r}-1}{\rho}$ and $g_{2}(r, \rho) \triangleq \frac{\rho^{2 r}-1}{\rho}$, the conditional pdf of $Z=\left|h_{s d}\right|^{2}+\left|h_{r d}\right|^{2}$ is calculated as follows, for $z \leq$ $g_{1}(r, \rho)$

$$
\begin{aligned}
f_{Z \mid B}(z) & =\int_{0}^{z} e^{-(z-x)} \frac{e^{-x}}{1-e^{-g_{1}(r, \rho)}} d x \\
& =\frac{z e^{-z}}{1-e^{-g_{1}(r, \rho)}}
\end{aligned}
$$

For $z>g_{1}(r, \rho)$, the conditional pdf of $Z=\left|h_{s d}\right|^{2}+\left|h_{r d}\right|^{2}$ is given by

$$
\begin{aligned}
f_{Z \mid B}(z) & =\int_{0}^{g_{1}(r, \rho)} e^{-(z-x)} \frac{e^{-x}}{1-e^{-g_{1}(r, \rho)}} d x \\
& =\frac{g_{1}(r, \rho) e^{-z}}{1-e^{-g_{1}(r, \rho)}} .
\end{aligned}
$$

The conditional probability of outage is calculated as follows:

$$
\begin{aligned}
& \mathbb{P}\left(\left\{\left|h_{s d}\right|^{2}+\left|h_{r d}\right|^{2}<g_{2}(r, \rho)\right\} \mid\left\{\left|h_{s d}\right|^{2}<g_{1}(r, \rho)\right\}\right) \\
& =\int_{0}^{g_{1}(r, \rho)} \frac{z e^{-z}}{1-e^{-g_{1}(r, \rho)}} d z+\int_{g_{1}(r, \rho)}^{g_{2}(r, \rho)} \frac{g_{1}(r, \rho) e^{-z}}{1-e^{-g_{1}(r, \rho)}} d z \\
& =\frac{1-e^{-g_{1}(r, \rho)}-g_{1}(r, \rho) e^{-g_{2}(r, \rho)}}{1-e^{-g_{1}(r, \rho)}} \\
& \doteq 1-\frac{\rho^{r-1} e^{-\rho^{2 r-1}}}{1-e^{-\rho^{r-1}}} \\
& \doteq \rho^{2 r-1} .
\end{aligned}
$$

Substituting (88) and (92) into (85), the conditional probability of outage is given by

$$
\begin{aligned}
P\left(\mathcal{O}_{2} \mid \mathcal{O}_{1}\right) & \doteq \rho^{(2 r-1)}+\rho^{(2 r-1)}\left(1-\rho^{(2 r-1)}\right) \\
& \doteq \rho^{(2 r-1)} .
\end{aligned}
$$

Using (81), (82), (83) and (93), the DMT of the orthogonal opportunistic DF relaying is given by

$$
d(r)=(1-r)^{+}+(1-2 r)^{+}
$$

\section{APPENDIX II \\ OpPORTUNISTIC AF ORTHOGONAL RELAYING OVER A Simple Relay CHANNEL}

The outage probability of the relay-assisted mode, given that the nonrelay mode is in outage is given by

$$
\begin{gathered}
\mathbb{P}\left(\mathcal{O}_{2} \mid \mathcal{O}_{1}\right) \\
=\mathbb{P}\left(\left\{\frac{1}{2} \log \left(1+\left|h_{s d}\right|^{2} \rho+f\left(\left|h_{s r}\right|^{2} \rho,\left|h_{r d}\right|^{2} \rho\right)\right)<r \log \rho\right\} \mid\right. \\
\left.\left\{\log \left(1+\left|h_{s d}\right|^{2} \rho\right)<r \log \rho\right\}\right) \\
=\mathbb{P}\left(\left\{\left|h_{s d}\right|^{2}+\frac{1}{\rho} f\left(\left|h_{s r}\right|^{2} \rho,\left|h_{r d}\right|^{2} \rho\right)<\frac{\rho^{2 r}-1}{\rho}\right\} \mid\right. \\
\left.\left\{\left|h_{s d}\right|^{2}<\frac{\rho^{r}-1}{\rho}\right\}\right) .
\end{gathered}
$$

At high SNR, (96) can be approximated by

$$
\begin{gathered}
\mathbb{P}\left(\mathcal{O}_{2} \mid \mathcal{O}_{1}\right)=\mathbb{P}\left(\left\{\left|h_{s d}\right|^{2}+\frac{\left|h_{s r}\right|^{2}\left|h_{r d}\right|^{2}}{\left|h_{s r}\right|^{2}+\left|h_{r d}\right|^{2}}<\frac{\rho^{2 r}-1}{\rho}\right\} \mid\right. \\
\left.\left\{\left|h_{s d}\right|^{2}<\frac{\rho^{r}-1}{\rho}\right\}\right)
\end{gathered}
$$

where $\frac{\left|h_{s r}\right|^{2}\left|h_{r d}\right|^{2}}{\left|h_{s r}\right|^{2}+\left|h_{r s}\right|^{2}}$ represents the harmonic mean of two independent exponential random variables. Using the result of [47], the harmonic mean of two exponential random variables with exponential parameters $\lambda$ can be approximated by an exponential random variable with exponential parameter $2 \lambda$.

In order to calculate the conditional outage probability distribution, we first calculate the conditional density function of $Z=\left|h_{s d}\right|^{2}+V$ where $V=\frac{\left|h_{s r}\right|^{2}\left|h_{r d}\right|^{2}}{\left|h_{s r}\right|^{2}+\left|h_{r s}\right|^{2}}$. Again, we are assuming $g_{1}(r, \rho)=\frac{\rho^{r}-1}{\rho}, g_{2}(r, \rho)=\frac{\rho^{2 r}-1}{\rho}$, and conditioning is over the event $B=\left\{h_{s d} \mid<\frac{\rho^{r}-1}{\rho}\right\}$. The conditional probability density function of $Z=\left|h_{s d}\right|^{2}+V$ is given by

$$
f_{Z \mid B}(z)= \begin{cases}\frac{2 e^{-2 z}\left(e^{z}-1\right)}{1-e^{-g_{1}(r, \rho)}}, & z \leq g_{1}(r, \rho) \\ \frac{2 e^{-2 z}\left(e^{g_{1}(r, \rho)}-1\right)}{1-e^{-g_{1}(r, \rho)}}, & z>g_{1}(r, \rho)\end{cases}
$$


The conditional probability of outage is calculated as follows:

$$
\begin{aligned}
\mathbb{P}\left(\left|h_{s d}\right|^{2}+\right. & \left.\left|h_{r d}\right|^{2}<\left.g_{2}(r, \rho)|| h_{s d}\right|^{2}<g_{1}(r, \rho)\right) \\
= & 2 \int_{0}^{g_{1}(r, \rho)} \frac{e^{-2 z}\left(e^{z}-1\right)}{1-e^{-g_{1}(r, \rho)}} d z \\
& \quad+2 \int_{g_{1}(r, \rho)}^{g_{2}(r, \rho)} \frac{e^{-2 z}\left(e^{g_{1}(r, \rho)}-1\right)}{1-e^{-g_{1}(r, \rho)}} d z \\
= & \frac{e^{-2 g_{2}(r, \rho)}-e^{-g_{1}(r, \rho)}-e^{-2 g_{2}(r, \rho)+g_{1}(r, \rho)}+1}{1-e^{-g_{1}(r, \rho)}} \\
\doteq & 1+e^{-2 \rho^{2 r-1}} \frac{1-e^{\rho^{r-1}}}{1-e^{-\rho^{r-1}}} \\
\doteq & \rho^{2 r-1} .
\end{aligned}
$$

Using (81), (82), (83), and (99), the DMT of the orthogonal opportunistic AF relaying is given by

$$
d(r)=(1-r)^{+}+(1-2 r)^{+} .
$$

\section{APPENDIX III \\ GENIE-AIDED DMT UPPER BOUND FOR THE SHARED RELAY CHANNEL}

The indexing of the access modes does not affect the problem; therefore, we can order the conditional events in Lemma 1, 2 arbitrarily. In the following, we index the outage events according to the order of selection that is described below, which is designed to sort out the dependencies in a way to make computations tractable.

The selection algorithm is as follows: If the nonrelayed configuration (shown in Fig. 8 part (c)) can support the required rate $R=r \log \rho$, it is selected. We shall call this Mode 1 in the remainder of Appendices. If Mode 1 is in outage (an event denoted by $\mathcal{U}_{1}$ ) we will check to see if either of the two direct links can individually support half the rate, i.e., $R=\frac{r}{2} \log \rho$. If one of the direct links can support this reduced rate, we consider the relayed mode sharing that direct link. (If none of the direct links can even support half the rate, we can consider either one at random.) This relayed mode shall be called Mode 2. If Mode 2 can support the full required rate, it is selected. The outage of Mode 2 is denoted $\mathcal{U}_{2}$. If both Modes 1, 2 are in outage, the remaining relayed mode, which will be denoted Mode 3 , is selected. The outage of Mode 3 is denoted $\mathcal{U}_{3}$ in this and the following appendices. The error events corresponding to the three modes are denoted $e_{1}^{\prime}, e_{2}^{\prime}, e_{3}^{\prime}$ in this and subsequent Appendices.

The total DMT of the genie-aided system is

$$
d(r)=d_{1}^{\prime}(r)+d_{2}^{\prime}(r)+d_{3}^{\prime}(r)
$$

where

$$
\begin{aligned}
& d_{1}^{\prime}(r)=-\lim _{\rho \rightarrow \infty} \frac{\log \mathbb{P}\left(e_{1}^{\prime}\right)}{\log \rho}, \\
& d_{2}^{\prime}(r)=-\lim _{\rho \rightarrow \infty} \frac{\log \mathbb{P}\left(e_{2}^{\prime} \mid e_{1}^{\prime}\right)}{\log \rho}, \\
& d_{3}^{\prime}(r)=-\lim _{\rho \rightarrow \infty} \frac{\log \mathbb{P}\left(e_{3}^{\prime} \mid e_{2}^{\prime}, e_{1}^{\prime}\right)}{\log \rho} .
\end{aligned}
$$

Although the expressions above are in terms of error events, in the remainder of this Appendix the diversities are expressed in terms of outage events instead of error events due to the fact that the genie-aided modes are equivalent to MISO channels and the codewords are assumed to be long enough.

Mode 1, access mode (c), represents a parallel Rayleigh channel. The outage of a parallel Rayleigh channel, $\mathbb{P}\left(\mathcal{O}_{3}\right)$, is given by

$$
\mathbb{P}\left(\mathcal{O}_{3}\right)=\mathbb{P}\left(\mathcal{O}_{31}\right)+\mathbb{P}\left(\mathcal{O}_{32}\right)+\mathbb{P}\left(\mathcal{O}_{33}\right)
$$

where $\mathcal{O}_{31}, \mathcal{O}_{32}$ and $\mathcal{O}_{33}$ partition the outage event $\mathcal{O}_{3}$ according to whether the first, the second, or both direct links are in outage

$$
\begin{aligned}
\mathbb{P}\left(\mathcal{O}_{31}\right)= & \mathbb{P}\left(\log \left(1+\left|h_{11}\right|^{2} \rho\right)<\frac{r}{2} \log \rho\right) \\
& \times \mathbb{P}\left(\log \left(1+\left|h_{22}\right|^{2}\right) \rho \geq \frac{r}{2} \log \rho\right) \\
\mathbb{P}\left(\mathcal{O}_{32}\right)= & \mathbb{P}\left(\log \left(1+\left|h_{11}\right|^{2} \rho\right) \geq \frac{r}{2} \log \rho\right) \\
& \times \mathbb{P}\left(\log \left(1+\left|h_{22}\right|^{2}\right) \rho<\frac{r}{2} \log \rho\right) \\
\mathbb{P}\left(\mathcal{O}_{33}\right)= & \mathbb{P}\left(\log \left(1+\left|h_{11}\right|^{2} \rho\right)<\frac{r}{2} \log \rho\right) \\
& \times \mathbb{P}\left(\log \left(1+\left|h_{22}\right|^{2}\right) \rho<\frac{r}{2} \log \rho\right) .
\end{aligned}
$$

Therefore, in the asymptote of high SNR

$$
P\left(\mathcal{O}_{3}\right) \doteq \rho^{r / 2-1}+\rho^{r / 2-1}+\rho^{r-2} \doteq \rho^{r / 2-1} .
$$

The unconditional DMT of the nonrelayed mode

$$
d_{1}^{\prime}(r)=\left(1-\frac{r}{2}\right)^{+} .
$$

To calculate $d_{2}^{\prime}(r)$ and $d_{3}^{\prime}(r)$, we study the outage of the respective access modes. We start by calculating the conditional outage of Mode 3 and use the result to calculate the conditional outage for Mode 2

$$
\begin{array}{r}
\mathbb{P}\left(\mathcal{U}_{3} \mid \mathcal{U}_{2}, \mathcal{U}_{1}\right)=\mathbb{P}\left(\left\{\log \left(1+\left(\left|h_{i i}\right|^{2}+\left|h_{r i}\right|^{2}\right) \rho\right)<r \log \rho\right\} \mid\right. \\
\left\{\left|h_{j j}\right|^{2}<f_{2}^{-1}\left(R,\left|h_{r, d_{j}}\right|^{2}\right)\right\},\left\{\left|h_{11}\right|^{2}<\frac{\rho^{r / 2}-1}{\rho}\right\} \\
\left.\left\{\left|h_{22}\right|^{2}<\frac{\rho^{r / 2}-1}{\rho}\right\}\right) \\
\doteq \mathbb{P}\left(\left\{\left|h_{i i}\right|^{2}+\left|h_{r i}\right|^{2}<\frac{\rho^{r}-1}{\rho}\right\} \mid\left\{\left|h_{i i}\right|^{2}<\frac{\rho^{r / 2}-1}{\rho}\right\}\right)
\end{array}
$$

where $i$ is the index of the source selected in Mode 3 and $j$ is the index of the source selected in Mode 2. The channel gain $\gamma_{i i} \triangleq\left|h_{i i}\right|^{2}$, conditioned on the event $B=\left\{\left|h_{i i}\right|<\frac{\rho^{r / 2}-1}{\rho}\right\}$ has the following conditional distribution

$$
f_{\gamma_{i i} B}(x)= \begin{cases}\frac{e^{-x}}{1-e^{-\frac{\rho^{r / 2}-1}{\rho}},} & x \leq \frac{\rho^{r / 2}-1}{\rho} \\ 0, & x>\frac{\rho^{r / 2}-1}{\rho} .\end{cases}
$$


Defining $g_{1}(r, \rho) \triangleq \frac{\rho^{r / 2}-1}{\rho}$ and $g_{2}(r, \rho) \triangleq \frac{\rho^{r}-1}{\rho}$, the condi- We notice that tional probability density function of $Z=\left|h_{i i}\right|^{2}+\left|h_{r i}\right|^{2}$ is

$$
f_{Z \mid B}(z)= \begin{cases}\frac{z e^{-\lambda z}}{1-e^{-g_{1}(r, \rho)}}, & z \leq g_{1}(r, \rho) \\ \frac{g_{1}(r, \rho) e^{-z}}{1-e^{-g_{1}(r, \rho)}}, & z>g_{1}(r, \rho) .\end{cases}
$$

The probability of outage $\mathbb{P}\left(\mathcal{U}_{3} \mid \mathcal{U}_{2}, \mathcal{U}_{1}\right)$ can be calculated as follows:

$$
\begin{aligned}
& \mathbb{P}\left(\mathcal{U}_{3} \mid \mathcal{U}_{2}, \mathcal{U}_{1}\right)= \int_{0}^{g_{1}(r, \rho)} \frac{z e^{-z}}{1-e^{-g_{1}(r, \rho)}} d z \\
&+\int_{g_{1}(r, \rho)}^{g_{2}(r, \rho)} \frac{g_{1}(r, \rho) e^{-z}}{1-e^{-g_{1}(r, \rho)}} d z \\
&= \frac{1-e^{-g_{1}(r, \rho)}-g_{1}(r, \rho) e^{-g_{2}(r, \rho)}}{1-e^{-g_{1}(r, \rho)}} \\
& \doteq 1-\frac{\rho^{r / 2-1} e^{-\rho^{r-1}}}{1-e^{-\rho^{r / 2-1}}} \doteq \rho^{r-1}
\end{aligned}
$$

To facilitate the analysis of the conditional outage of Mode 2 , we introduce a partition of $\mathcal{U}_{1}$. Define $\mathcal{V}$ as the event that $a t$ least one of the direct links can support half the desired rate, i.e., $\frac{r}{2} \log \rho$, and introduce

$$
\mathcal{V}_{1}=\mathcal{V} \cap \mathcal{U}_{1} \quad \mathcal{V}_{2}=\overline{\mathcal{V}} \cap \mathcal{U}_{1}
$$

Thus, $\mathcal{V}_{1}$ is the event that the nonrelayed Mode 1 is in outage, and yet at least one of the two direct links can support at least half the desired rate, i.e., $\frac{r}{2} \log \rho$

$$
\begin{aligned}
\mathbb{P}\left(\mathcal{U}_{2} \mid \mathcal{U}_{1}\right) & =\frac{\mathbb{P}\left(\mathcal{U}_{2}, \mathcal{U}_{1}\right)}{\mathbb{P}\left(\mathcal{U}_{1}\right)} \\
& =\frac{\mathbb{P}\left(\mathcal{U}_{2} \mid \mathcal{V}_{1}\right) \mathbb{P}\left(\mathcal{V}_{1}\right)+\mathbb{P}\left(\mathcal{U}_{2} \mid \mathcal{V}_{2}\right) \mathbb{P}\left(\mathcal{V}_{2}\right)}{\mathbb{P}\left(\mathcal{V}_{1}\right)+\mathbb{P}\left(\mathcal{V}_{2}\right)} \\
& \doteq \frac{\rho^{2(r-1)} 2 \rho^{\left(\frac{r}{2}-1\right)}+\rho^{(r-1)} \rho^{2\left(\frac{r}{2}-1\right)}}{2 \rho^{\left(\frac{r}{2}-1\right)}+\rho^{2\left(\frac{r}{2}-1\right)}} \\
& \doteq \rho^{2(r-1)}
\end{aligned}
$$

where $\mathbb{P}\left(\mathcal{V}_{1}\right)=\mathbb{P}\left(\mathcal{O}_{31}\right)+\mathbb{P}\left(\mathcal{O}_{32}\right) \doteq 2 \rho^{(r / 2-1)}$ from (106) and (107), $\mathbb{P}\left(\mathcal{V}_{2}\right)=\mathbb{P}\left(\mathcal{O}_{33}\right) \doteq \rho^{2(r / 2-1)}$ from (108). The probability of $U_{2}$ conditioned on $\mathcal{V}_{2}$ is equivalent to (111) and, hence, $\mathbb{P}\left(\mathcal{U}_{2} \mid \mathcal{V}_{2}\right) \doteq \rho^{(r-1)}$. The conditional probability $\mathbb{P}\left(\mathcal{U}_{2} \mid \mathcal{V}_{1}\right)$ is given by

$$
\begin{aligned}
& \mathbb{P}\left(\mathcal{U}_{2} \mid \mathcal{V}_{1}\right) \\
& \quad=\mathbb{P}\left(\left\{\left|h_{i i}\right|^{2}+\left|h_{r i}\right|^{2}<\frac{\rho^{r}-1}{\rho}\right\} \mid\left\{\left|h_{i i}\right|^{2}>\frac{\rho^{r / 2}-1}{\rho}\right\}\right) .
\end{aligned}
$$

$$
\begin{aligned}
& \mathbb{P}\left(\left\{\left|h_{i i}\right|^{2}+\left|h_{r i}\right|^{2}<\frac{\rho^{r}-1}{\rho}\right\}\right)= \\
& \mathbb{P}\left(\left\{\left|h_{i i}\right|^{2}+\left|h_{r i}\right|^{2}<\frac{\rho^{r}-1}{\rho}\right\} \mid\left\{\left|h_{i i}\right|^{2}<\frac{\rho^{r / 2}-1}{\rho}\right\}\right) \\
& \quad \times \mathbb{P}\left(\left\{\left|h_{i i}\right|^{2}<\frac{\rho^{r / 2}-1}{\rho}\right\}\right) \\
& \quad+\mathbb{P}\left(\left\{\left|h_{i i}\right|^{2}+\left|h_{r i}\right|^{2}<\frac{\rho^{r}-1}{\rho}\right\} \mid\left\{\left|h_{i i}\right|^{2}>\frac{\rho^{r / 2}-1}{\rho}\right\}\right) \\
& \quad \times \mathbb{P}\left(\left\{\left|h_{i i}\right|^{2}>\frac{\rho^{r / 2}-1}{\rho}\right\}\right) .
\end{aligned}
$$

At high SNR, using result from (114), (118) leads to

$$
\begin{aligned}
\rho^{2(r-1)} \doteq \rho^{(r-1)} \rho^{(r / 2-1)}+\mathbb{P} & \left(\left\{\left|h_{i i}\right|^{2}+\left|h_{r i}\right|^{2}<\frac{\rho^{r}-1}{\rho}\right\} \mid\right. \\
& \left.\left\{\left|h_{i i}\right|^{2}>\frac{\rho^{r / 2}-1}{\rho}\right\}\right)
\end{aligned}
$$

where the random variable $\left|h_{i i}\right|^{2}+\left|h_{r i}\right|^{2}$ has Gamma distribution. Using (117) and (119), one can see that $\mathbb{P}\left(\mathcal{U}_{2} \mid \mathcal{V}_{1}\right)=$ $\rho^{2(r-1)}$.

Equations (116) and (114) indicate that

$$
\begin{aligned}
& d_{2}^{\prime}(r)=2(1-r)^{+} \\
& d_{3}^{\prime}(r)=(1-r)^{+} .
\end{aligned}
$$

The DMT of the genie aided system is given by

$$
\begin{aligned}
d(r) & =\left(1-\frac{r}{2}\right)^{+}+2(1-r)^{+}+(1-r)^{+} \\
& = \begin{cases}4-\frac{7}{2} r, & 0 \leq r \leq 1 \\
\left(1-\frac{r}{2}\right), & 1<r \leq 2 .\end{cases}
\end{aligned}
$$

\section{APPENDIX IV}

\section{NAF ACHIEVABLE DMT FOR THE SHARED Relay CHANNEL}

The DMT of the NAF protocol for the shared relay channel will be calculated according to the selection algorithm developed in Appendix III, which we invite the reader to review before continuing with the present Appendix.

The overall diversity is governed by (101), and we need to calculate $d_{1}^{\prime}(r), d_{2}^{\prime}(r), d_{3}^{\prime}(r)$.

To begin with, the DMT of the nonrelayed mode does not depend on the relaying protocol, so there is no need to calculate it again: it is $d_{1}^{\prime}(r)=\left(1-\frac{r}{2}\right)^{+}$as calculated in expression (110). 
For calculating $d_{3}^{\prime}(r)$, the equivalence of error and outage analysis is nontrivial and will be relegated to Appendix V. In this Appendix we analyze the conditional outage of Mode 3

$$
\begin{aligned}
\mathbb{P}\left(\mathcal{U}_{3} \mid \mathcal{U}_{2}, \mathcal{U}_{1}\right) \doteq & \frac{1}{2} \mathbb{P}\left(I_{1}<R \mid \overline{\mathcal{V}}, I_{2}<R\right) \\
& +\frac{1}{2} \mathbb{P}\left(I_{2}<R \mid \overline{\mathcal{V}}, I_{1}<R\right) \\
\doteq & \mathbb{P}\left(I_{1}<R \mid\left\{\left|h_{11}\right|^{2}<\frac{\rho^{r / 2}-1}{\rho}\right\}\right)
\end{aligned}
$$

where $I_{1}$ and $I_{2}$ are the instantaneous mutual information of the simple relay channel for User 1 and User 2, respectively. Symmetry arguments have been used to simplify the expression. We will use the exponential order of channel gains, defined thus

$$
v=-\lim _{\rho \rightarrow \infty} \frac{\log |h|^{2}}{\log \rho}
$$

where $v$ itself is a random variable. Recall that the conditional pdf of the source-destination channel gain $\left|h_{11}\right|^{2}$, subject to $h_{11}$ not supporting rate $\frac{r}{2} \log \rho$, is given by (112). The exponential order of this conditional random variable is denoted $v_{1}$ whose pdf can be calculated as follows

$$
f\left(v_{1}\right)= \begin{cases}\ln \rho \rho^{-v_{1}} \frac{e^{-\rho^{-v_{1}}}}{1-e^{-\frac{\rho^{r / 2}-1}{\rho}},}, & v_{1} \geq 1-\frac{r}{2} \\ 0, & v_{1}<1-\frac{r}{2} .\end{cases}
$$

As $\rho \rightarrow \infty$ we can show that

$$
f\left(v_{1}\right) \doteq \begin{cases}\rho^{-v_{1}-(r / 2-1)}, & v_{1} \geq 1-\frac{r}{2} \\ 0, & v_{1}<1-\frac{r}{2}\end{cases}
$$

Also, the channel gains $\left|h_{r 1}\right|^{2}$ and $\left|h_{1 r}\right|^{2}$ (exponentially distributed, unconditioned) have exponential orders that are denoted $v_{2}$ and $v_{3}$, respectively. Furthermore, the pdf of $v_{1}, v_{2}, v_{3}$ are in turn characterized by their asymptotic exponential orders $f\left(v_{i}\right) \doteq \rho^{-u_{i}}$, over their respective regions of support.

In a manner similar to [28], the outage region is more conveniently addressed in the space of the exponential orders, i.e.,

$$
O=\left\{\left(v_{1}, v_{2}, v_{3}\right): I<r \log \rho\right\}
$$

We can now calculate

$$
\begin{aligned}
\mathbb{P}(I<r & \log \rho)=\iiint_{O} f\left(v_{1}, v_{2}, v_{3}\right) d v_{1} d v_{2} d v_{3} \\
= & \iiint_{O^{\prime}} \log \rho \rho^{-v_{1}} \frac{e^{-\rho^{-v_{1}}}}{1-e^{-\frac{\rho^{r / 2}-1}{\rho}}} \log \rho \rho^{-v_{2}} e^{-\rho^{-v_{2}}} \\
& \quad \times \log \rho \rho^{-v_{3}} e^{-\rho^{-v_{3}} d v_{1} d v_{2} d v_{3}} \\
\doteq & \iiint_{O^{\prime}} \rho^{-\sum u_{i}} d v_{1} d v_{2} d v_{3} \\
\doteq & \rho^{-d_{o}}
\end{aligned}
$$

where $O^{\prime}$ is the intersection of $O$ and the support of $f\left(v_{1}, v_{2}, v_{3}\right)$, and

$$
\begin{aligned}
d_{o} & =\inf _{\left(v_{1}, v_{2}, v_{3}\right) \in O^{\prime}} \sum_{j=1}^{n} u_{i} \\
& =\inf _{\left(v_{1}, v_{2}, v_{3}\right) \in O^{\prime}} v_{1}+(r / 2-1)+v_{2}+v_{3} .
\end{aligned}
$$

Following the same steps as those used in the proof of $[28$, Theorem 2]

$$
\begin{aligned}
O^{\prime}= & \left\{\left(v_{1}, v_{2}, v_{3}\right) \in R^{3+}, v_{1} \geq\left(1-\frac{r}{2}\right),\right. \\
& {\left[\max \left(\left(1-v_{1}\right), \frac{1}{2}\left(1-\left(v_{2}+v_{3}\right)\right)\right]^{+}<r\right\} . }
\end{aligned}
$$

Solving (130), we can show that

$$
d_{0}=(1-2 r)^{+} .
$$

It remains to show that $d_{3}^{\prime}(r)=d_{0}$, which will be done in Appendix V.

For calculating $d_{2}^{\prime}(r)$, we follow steps essentially similar to those leading to (116), except this time we need to make explicit the relationship between outage and error events

$$
\begin{aligned}
\mathbb{P}\left(e_{2}^{\prime} \mid e_{1}^{\prime}\right) & \doteq \mathbb{P}\left(e_{2}^{\prime} \mid \mathcal{U}_{1}\right) \\
& =\frac{\mathbb{P}\left(e_{2}^{\prime}, \mathcal{U}_{1}\right)}{\mathbb{P}\left(\mathcal{U}_{1}\right)} \\
& =\frac{\mathbb{P}\left(e_{2}^{\prime} \mid \mathcal{V}_{1}\right) \mathbb{P}\left(\mathcal{V}_{1}\right)+\mathbb{P}\left(e_{2}^{\prime} \mid \mathcal{V}_{2}\right) \mathbb{P}\left(\mathcal{V}_{2}\right)}{\mathbb{P}\left(\mathcal{V}_{1}\right)+\mathbb{P}\left(\mathcal{V}_{2}\right)} \\
& \doteq \frac{\rho^{(r-1)} \rho^{(2 r-1)} 2 \rho^{\left(\frac{r}{2}-1\right)}+\rho^{(2 r-1)} \rho^{2\left(\frac{r}{2}-1\right)}}{2 \rho^{\left(\frac{r}{2}-1\right)}+\rho^{2\left(\frac{r}{2}-1\right)}} \\
& \doteq \rho^{-(1-r)^{+}-(1-2 r)^{+}} .
\end{aligned}
$$

where (133) is true because $e_{1}^{\prime}$ is the error of a nonrelayed link; therefore, with long codewords, it is exponentially equivalent to the outage event $\mathcal{U}_{1}$. Equation (134) is derived by substituting the known error exponents and noting that the third term is dominated by the first two in both the numerator and denominator. Overall, $d_{2}^{\prime}(r)=(1-r)^{+}+(1-2 r)^{+}$can be obtained.

To summarize, we have calculated $d_{1}^{\prime}(r), d_{2}^{\prime}(r)$ and $d_{3}(r)$.

\section{APPENDIX V \\ RELATION OF OUTAGE AND ERROR EVENTS FOR THE SHARED RELAY CHANNEL}

In this Appendix, we show that the outage and error events have the same exponential order. The approach follows [28, Theorem 3] and is adapted to the specific case at hand. We need 
to show $\mathbb{P}(e) \dot{\leq} \mathbb{P}(\mathcal{O})$ and $\mathbb{P}(e) \dot{\geq} \mathbb{P}(\mathcal{O})$. The former is a straightforward application of [18, Lemma 5]. For showing the latter inequality, note that

$$
\begin{aligned}
\mathbb{P}(e) & =\mathbb{P}(\mathcal{O}) \mathbb{P}(e \mid \mathcal{O})+\mathbb{P}(e, \overline{\mathcal{O}}) \\
& \leq \mathbb{P}(\mathcal{O})+\mathbb{P}(e, \overline{\mathcal{O}}) \\
& \doteq \mathbb{P}(\mathcal{O})
\end{aligned}
$$

where the last equation is valid whenever $\mathbb{P}(e, \overline{\mathcal{O}}) \dot{\leq} \mathbb{P}(\mathcal{O})$, whose verification is the subject of the remainder of this Appendix. The pairwise error probability conditioned on the channel coefficients is given by

$$
P_{\mathbf{c} \rightarrow \mathbf{e} \mid h_{s d}, h_{s r}, h_{r d}} \leq \operatorname{det}\left(\mathbf{I}_{2}+\frac{1}{2} \boldsymbol{\Sigma}_{s} \boldsymbol{\Sigma}_{n}^{-1}\right)^{-\ell / 2}
$$

where $\ell$ is the codebook codeword length and $\boldsymbol{\Sigma}_{s}$ and $\boldsymbol{\Sigma}_{n}$ are the covariance matrices of the received signal and the noise, respectively. The pair wise error probability is given by

$$
P_{\mathbf{c} \rightarrow \mathbf{e} \mid v_{1}, v_{2}, v_{3}} \dot{\leq} \rho^{-\frac{l}{2} \max \left(2\left(1-v_{1}\right), 1-\left(v_{2}+v_{3}\right)\right)^{+}}
$$

where

$$
\left(v_{1}, v_{2}, v_{3}\right) \in R^{3+} \cap\left\{v_{1} \geq\left(1-\frac{r}{2}\right)\right\} .
$$

The total probability of error is

$$
P_{e \mid v_{1}, v_{2}, v_{3}} \dot{\leq} \rho^{-\frac{l}{2}\left(\left[\max \left(2\left(1-v_{1}\right), 1-\left(v_{2}+v_{3}\right)\right)\right]^{+}-2 r\right)} .
$$

The probability of error while no outage $\mathbb{P}(e, \overline{\mathcal{O}})$ satisfies (141), shown at the bottom of the page, where $O^{\prime \prime}=\left\{\left(v_{1}, v_{2}, v_{3}\right) \in\right.$ $\left.R^{+}:\left(v_{1}, v_{2}, v_{3}\right) \notin O^{\prime}\right\}$, the area in the positive quadrant that is the complement of $O^{\prime}$. Recall that $O^{\prime}$ is the outage region in the space of exponents, as defined in (131). The integral is dominated by the minimum value of the SNR exponent over $\overline{\mathcal{O}}$, i.e.,

$$
\mathbb{P}(e, \overline{\mathcal{O}}) \dot{\leq} \rho^{-d_{1}(r)}
$$

where

$$
\begin{aligned}
& d_{1}(r)= \\
& \inf _{v_{1}, v_{2}, v_{3} \in O^{\prime \prime}}\left\{\begin{array}{l} 
\\
\quad
\end{array}\right. \\
& \left.\quad+v_{1}+(r / 2-1)+v_{2}+v_{3}\right\} .
\end{aligned}
$$

Note that the multiplier of $\ell$ is positive throughout the region $O^{\prime \prime}$. Now recall from the previous Appendix that the outage probability is

$$
\mathbb{P}(\mathcal{O}) \doteq \rho^{-d_{0}(r)}
$$

where

$$
d_{0}(r)=\inf _{\left(v_{1}, v_{2}, v_{3}\right) \in O^{\prime}} v_{1}+(r / 2-1)+v_{2}+v_{3} .
$$

The expression for $d_{1}(r)$ has one extra term compared with $d_{0}(r)$ which, as mentioned above, is positive and can be made as large as desired by choosing $\ell$ to be large enough. Therefore, the condition $\mathbb{P}(e, \overline{\mathcal{O}}) \leq \mathbb{P}(\mathcal{O})$ is established, leading to $\mathbb{P}(e) \leq \mathbb{P}(\mathcal{O})$, which completes the proof that the probability of error and outage events are exponentially equivalent.

\section{APPENDIX VI}

\section{DMT FOR DDF OPPORTUNISTIC SHARED RELAY CHANNEL}

We derive an achievable DMT for the DDF opportunistic shared relay channel, employing the mode selection rule defined in Appendix III. The DMT is given by (101), (102), (103) and (104). The reader is referred to Appendix III for the definition of the access modes as well as the selection rule.

The DMT for Mode 1 is not affected by the relay and is given by $d_{1}^{\prime}(r)=(1-r / 2)^{+}$, as seen in previous appendices. For Mode 2 one can employ the techniques of Appendix III to show that outage is dominated by the event of one link being in outage; hence, using results from [28], one can prove that

$$
d_{2}^{\prime}(r)= \begin{cases}2(1-r), & 0 \leq r \leq \frac{1}{2} \\ \frac{1-r}{r}, & \frac{1}{2} \leq 1 .\end{cases}
$$

To calculate $d_{3}^{\prime}(r)$, we consider the conditional outage of Mode 3; the equivalence of error and outage analysis can be shown in a manner similar to Appendix V and [28], and is omitted for brevity. In the following we directly derive diversity from the outage events. The conditional outage of Mode 3 was calculated in (124)

$$
\mathbb{P}\left(\mathcal{U}_{3} \mid \mathcal{U}_{2}, \mathcal{U}_{1}\right) \doteq \mathbb{P}\left(I_{1}<R \mid\left\{\left|h_{11}\right|^{2}<\frac{\rho^{r / 2}-1}{\rho}\right\}\right) .
$$

Given that $\left|h_{11}\right|^{2}<\frac{\rho^{r / 2}-1}{\rho}$, the exponential order of $\left|h_{11}\right|^{2}$ is proved in (127) to have the following distribution at high SNR:

$$
f\left(v_{i}\right) \doteq \begin{cases}\rho^{-v_{i}-(r / 2-1)}, & v_{i} \geq 1-\frac{r}{2} \\ 0, & v_{i}<1-\frac{r}{2}\end{cases}
$$

$$
\begin{aligned}
\mathbb{P}(e, \overline{\mathcal{O}}) & \leq \iiint_{O^{\prime \prime}} P_{e \mid v_{1}, v_{2}, v_{3}} \mathbb{P}\left(\left(v_{1}, v_{2}, v_{3}\right) \in \overline{\mathcal{O}}\right) d v_{1} d v_{2} d v_{3} \\
& =\iiint_{O^{\prime \prime}} \rho^{-\frac{l}{2}\left(\left[\max \left(2\left(1-v_{1}\right), 1-\left(v_{2}+v_{3}\right)\right)\right]^{+}-2 r\right)+v_{1}+\left(\frac{r}{2}-1\right)+v_{2}+v_{3}} d v_{1} d v_{2} d v_{3}
\end{aligned}
$$


The outage as shown in (129) is given by

$$
\mathbb{P}\left(\mathcal{U}_{3} \mid \mathcal{U}_{2}, \mathcal{U}_{1}\right) \doteq \rho^{-d_{3}^{\prime}(r)}
$$

where

$$
d_{3}^{\prime}(r)=\inf _{\left(v_{1}, v_{2}, v_{3}\right) \in O^{\prime}} v_{1}+(r / 2-1)+v_{2}+v_{3} .
$$

Following the same steps as the proof of [28, Theorem 5], the outage event $O^{\prime}$ is defined as

$$
\begin{aligned}
& O^{\prime}=\left\{\left(v_{1}, v_{2}, v_{3}\right) \in R^{3+}, v_{1} \geq(1-r / 2)\right. \\
& \left.\quad t\left(1-v_{1}\right)^{+}+(1-t)\left(1-\min \left(v_{1}, v_{2}\right)\right)^{+} \leq r\right\}
\end{aligned}
$$

where $t$ is the listening-time ratio of the half-duplex relay, with $r \leq t \leq 1$.

To get the DMT, we need to solve the optimization problem of (147), (149). Solving the above optimizations and combining the results, the DMT is given by

$$
d_{3}^{\prime}(r)= \begin{cases}1-\frac{r}{1-r}\left(1-\frac{r}{2}\right), & 0 \leq r \leq 0.5 \\ \frac{(1-r)}{r}-\left(1-\frac{r}{2}\right), & 0.5<r \leq 2-\sqrt{2} \\ 0, & 2-\sqrt{2}<r \leq 1 .\end{cases}
$$

Adding $d_{1}^{\prime}(r), d_{2}^{\prime}(r)$ and $d_{3}^{\prime}(r)$ completes the proof.

\section{APPENDIX VII}

\section{DMT FOR CF OpPORTUNISTIC SHARED RELAY CHANNEL}

The methods of this Appendix closely follow [30], with the notable exception of implementing the effects of our selection algorithm and the dependence between the nodes.

We use the selection criterion defined in Appendix III, and the DMT is given by (101), (102), (103) and (104). The DMT of nonrelayed Mode 3 is given by $d_{1}^{\prime}(r)=(1-r / 2)^{+}$, as seen several times already, since it is not contingent on the relay protocol.

To calculate $d_{2}^{\prime}(r)$ and $d_{3}^{\prime}(r)$, we borrow the following result from [30]. For the random half-duplex single-antenna relay channel, the dynamic-state CF protocol is DMT optimal and by random here we mean that the random binary state of the relay (listen/transmit) is used as a channel input and used in designing codebooks to convey information through the state of the relay.

For Mode 2, one can employ the techniques of Appendix III to show that outage is dominated by the event of one link being in outage; hence, using results from [30], one can prove that

$$
d_{2}^{\prime}(r)=2(1-r)^{+} .
$$

For Mode 3, the DMT is given by

$$
d_{3}^{\prime}(r)=\max _{t} \min \left(d_{M A C}(r, t), d_{B C}(r, t)\right)
$$

where

$$
\begin{aligned}
d_{B C} & =-\lim _{\rho \rightarrow \infty} \frac{\log \min _{p\left(x_{s}, x_{r} \mid q\right)} \mathbb{P}\left(I_{B C}<r \log \rho \mid \mathcal{U}_{2}, \mathcal{U}_{1}\right)}{\log \rho} \\
d_{M A C} & =-\lim _{\rho \rightarrow \infty} \frac{\log \min _{p\left(x_{s}, x_{r} \mid q\right)} \mathbb{P}\left(I_{M A C}<r \log \rho \mid \mathcal{U}_{2}, \mathcal{U}_{1}\right)}{\log \rho},
\end{aligned}
$$

where $q$ represents the state of the relay (listening versus transmitting), $p\left(x_{s}, x_{r} \mid q\right)$ is the probability density of the codebooks generated for the source and the relay, and $I_{B C}$ and $I_{M A C}$ represent the total mutual information across the source cutset and the destination cutset, respectively. It can be shown [30] that

$$
\begin{aligned}
I_{B C} \leq & (1-t) \log \left(1+\left(\left|h_{s^{*} d^{*}}\right|^{2}+\left|h_{s^{*} r}\right|^{2}\right) \rho\right) \\
& +t \log \left(1+\left|h_{s^{*} d^{*}}\right|^{2} \rho\right) \\
I_{M A C} \leq & (1-t) \log \left(1+\left|h_{s^{*} d^{*}}\right|^{2} \rho\right) \\
& +t \log \left(1+\left(\left|h_{s^{*} d^{*}}\right|^{2}+\left|h_{r d^{*}}\right|^{2}\right) \rho\right)
\end{aligned}
$$

where $s^{*}$ and $d^{*}$ are the selected source and destination for Mode 3. Using the same technique as in Appendix VI, we have

$$
\mathbb{P}\left(I_{B C}<r \log \rho \mid \mathcal{U}_{2}, \mathcal{U}_{1}\right) \doteq \rho^{-d_{B C}(r)}
$$

where

$$
d_{B C}(r)=\inf _{\left(v_{1}, v_{3}\right) \in O^{\prime}} v_{1}+(r / 2-1)+v_{3}
$$

and the outage event $O^{\prime}$ is defined as

$$
\begin{aligned}
O^{\prime}=\{ & \left(v_{1}, v_{3}\right) \in R^{2+}, v_{1} \geq(1-r / 2) \\
& \left.(1-t)\left(1-v_{1}\right)^{+}+t\left(1-\min \left(v_{1}, v_{3}\right)\right)^{+} \leq r\right\} .
\end{aligned}
$$

Solving the optimization problem, the DMT for the source cutset is given by

$$
d_{B C}= \begin{cases}1-\frac{r}{t}\left(1-\frac{1-t}{2}\right) & t>\frac{1}{2}, r \leq \frac{1-(1-t)}{1-(1-t) / 2} \\ 0 & t>\frac{1}{2}, r>\frac{1-(1-t)}{1-(1-t) / 2} \\ 1-r\left(\frac{1}{t}-\frac{1}{2}\right) & t \leq \frac{1}{2}, r \leq t \\ \frac{1-r}{1-t}+\frac{r}{2}-1 & t \leq \frac{1}{2}, \frac{1-(1-t)}{1-(1-t) / 2} \geq r>t \\ 0 & t \leq \frac{1}{2}, r>\frac{1-(1-t)}{1-(1-t) / 2} .\end{cases}
$$

Similarly, The DMT for the destination cutset is given by

$$
d_{M A C}= \begin{cases}1-\frac{r}{1-t}\left(1-\frac{t}{2}\right) & t<\frac{1}{2}, r \leq \frac{1-t}{1-t / 2} \\ 0 & t<\frac{1}{2}, r>\frac{1-t}{1-t / 2} \\ 1-r\left(\frac{1}{1-t}-\frac{1}{2}\right) & t \geq \frac{1}{2}, r \leq(1-t) \\ \frac{1-r}{t}+\frac{r}{2}-1 & t \geq \frac{1}{2}, \frac{1-t}{1-t / 2} \geq r>(1-t) \\ 0 & t \geq \frac{1}{2}, r>\frac{1-t}{1-t / 2} .\end{cases}
$$


The two functions are equal at $t=\frac{1}{2}$ and that gives the maximum DMT. The DMT is given by

$$
d_{3}^{\prime}(r)=\left(1-\frac{3}{2} r\right)^{+}
$$

Adding the DMT of the three modes completes the proof.

\section{REFERENCES}

[1] R. Knopp and P. A. Humblet, "Information capacity and power control in single-cell multiuser communications," in Proc. Int. Conf. Communications, Seattle, WA, Jun. 1995, pp. 331-335.

[2] T. E. Hunter and A. Nosratinia, "Distributed protocols for user cooperation in multi-user wireless networks," in Proc. IEEE Global Telecommunications Conf., Nov./Dec. 2004, vol. 6, pp. 3788-3792.

[3] A. Bletsas, A. Khisti, D. P. Reed, and A. Lippman, "A simple cooperative diversity method based on network path selection," IEEE J. Sel. Areas Commun., vol. 24, no. 3, pp. 659-672, Mar. 2006.

[4] A. Bletsas, H. Shin, and M. Z. Win, "Cooperative communications with outage-optimal opportunistic relaying," IEEE Trans. Wireless Commun., vol. 6, no. 9, pp. 3450-3460, Sep. 2007.

[5] A. Bletsas, H. Shin, and M. Z. Win, "Outage optimality of opportunistic amplify-and-forward relaying," IEEE Commun. Lett., vol. 11, no. 3, pp. 261-263, Mar. 2007.

[6] Y. Jing and H. Jafarkhani, "Single and multiple relay selection schemes and their achievable diversity orders," IEEE Trans. Wireless Commun., vol. 8, no. 3, pp. 1414-1423, Mar. 2009.

[7] I. Krikidis, J. Thompson, S. McLaughlin, and N. Goertz, "Amplifyand-forward with partial relay selection," IEEE Commun. Lett., vol. 12, no. 4, pp. 235-237, Apr. 2008.

[8] I. Krikidis, J. Thompson, S. Mclaughlin, and N. Goertz, "Max-min relay selection for legacy amplify-and-forward systems with interference," IEEE Trans. Wireless Commun., vol. 8, no. 6, pp. 3016-3027, Jun. 2009.

[9] R. Vaze and R. W. Heath, "To code in space and time or not in multihop relay channels," IEEE Trans. Signal Process., vol. 57, no. 7, pp. 2736-2747, Jul. 2009.

[10] A. Nosratinia and T. E. Hunter, "Grouping and partner selection in cooperative wireless networks," IEEE J. Sel. Areas Commun., vol. 25, no. 2, pp. 369-378, Feb. 2007

[11] M. M. Fareed and M. Uysal, "On relay selection for decode-and-forward relaying," IEEE Trans. Wireless Commun., vol. 8, no. 7, pp. 3341-3346, Jul. 2009.

[12] E. Beres and R. Adve, "Selection cooperation in multi-source cooperative networks," IEEE Trans. Wireless Commun., vol. 7, no. 1, pp. 118-127, Jan. 2008.

[13] S. Lee, M. Han, and D. Hong, "Average SNR and ergodic capacity analysis for opportunistic DF relaying with outage over Rayleigh fading channels," IEEE Trans. Wireless Commun., vol. 8, no. 6, pp. 2807-2812, Jun. 2009.

[14] T. J. Oechtering and H. Boche, "Bidirectional regenerative half-duplex relaying using relay selection," IEEE Trans. Wireless Commun., vol. 7, no. 5, pp. 1879-1888, May 2008.

[15] Z. Yi and I.-M. Kim, "Diversity order analysis of the decode-and-forward cooperative networks with relay selection," IEEE Trans. Wireless Commun., vol. 7, no. 5, pp. 1792-1799, May 2008.

[16] K. S. Hwang, Y. C. Ko, and M. S. Alouini, "Outage probability of cooperative diversity systems with opportunistic relaying based on decode-and-forward," IEEE Trans. Wireless Commun., vol. 7, no. 12, pp. 5100-5107, Dec. 2008

[17] R. Tannious and A. Nosratinia, "The interference channel with MIMO relay: Degrees of freedom," in Proc. IEEE Int. Symp. Information Theory, 2008, pp. 1908-1912.

[18] L. Zheng and D. N. C. Tse, "Diversity and multiplexing: A fundamental tradeoff in multiple-antenna channels," IEEE Trans. Inf. Theory, vol. 49, no. 5, pp. 1073-1096, May 2003.

[19] D. N. C. Tse, P. Viswanath, and L. Zheng, "Diversity-multiplexing tradeoff in multiple-access channels," IEEE Trans. Inf. Theory, vol. 50, no. 9, pp. 1859-1874, Sep. 2004.

[20] J. Laneman, D. Tse, and G. Wornell, "Cooperative diversity in wireless networks: Efficient protocols and outage behavior," IEEE Trans. Inf. Theory, vol. 50, no. 12, pp. 3062-3080, Dec. 2004.
[21] A. Carleial, "Interference channels," IEEE Trans. Inf. Theory, vol. 24, no. 1 , pp. 60-70, Jan. 1978.

[22] H. Sato, "The capacity of the Gaussian interference channel under strong interference (corresp.)," IEEE Trans. Inf. Theory, vol. 27, no. 6, pp. 786-788, Nov. 1981.

[23] O. Sahin and E. Erkip, "Achievable rates for the Gaussian interference relay channel," in Proc. IEEE Global Telecommunications Conf., 2007, pp. $1627-1631$.

[24] S. Sridharan, S. Vishwanath, S. Jafar, and S. Shamai, "On the capacity of cognitive relay assisted Gaussian interference channel," in Proc. IEEE Int. Symp. Information Theory, 2008, pp. 549-553.

[25] I. Maric, R. Dabora, and A. Goldsmith, "On the capacity of the interference channel with a relay," in Proc. IEEE Int. Symp. Information Theory, 2008, pp. 554-558.

[26] M. O. Hasna and M. S. Alouini, "End-to-end performance of transmission systems with relays over Rayleigh-Fading channels," IEEE Trans. Wireless Commun., vol. 2, no. 6, pp. 1126-1131, Nov. 2003.

[27] R. U. Nabar, H. Bolcskei, and F. W. Kneubuhler, "Fading relay channels: Performance limits and space-time signal design," IEEE J. Sel. Areas Commun., vol. 22, no. 6, pp. 1099-1109, Aug. 2004.

[28] K. Azarian, H. El Gamal, and P. Schniter, "On the achievable diversity-multiplexing tradeoff in half-duplex cooperative channels," IEEE Trans. Inf. Theory, vol. 51, no. 12, pp. 4152-4172, Dec. 2005.

[29] M. Abouelseoud and A. Nosratinia, "Diversity multiplexing trade-off for opportunistic multiple access relay channel," presented at the Asilomar Conf. Signals, Systems and Computers, 2008.

[30] M. Yuksel and E. Erkip, "Multiple-antenna cooperative wireless systems: A diversity multiplexing tradeoff perspective," IEEE Trans. Inf. Theory, vol. 53, no. 10, pp. 3371-3393, Oct. 2007.

[31] G. Kramer, M. Gastpar, and P. Gupta, "Cooperative strategies and capacity theorems for relay networks," IEEE Trans. Inf. Theory, vol. 51, no. 9, pp. 3037-3063, Sep. 2005.

[32] S. Pawar, A. Avestimehr, and D. N. C. Tse, "Diversity-multiplexing tradeoff of the half-duplex relay channel," in Proc. 46th Annu. Allerton Conf. Communication, Control and Computing, 2008, pp. 27-34.

[33] D. Gunduz, A. Glodsmith, and V. Poor, "MIMO two-way relay channel: Diversity-multiplexing tradeoff analysis," presented at the Asilomar Conf. Signals, Systems and Computers, Pasific Grove, CA, 2008.

[34] A. Tajer and A. Nosratinia, "A broadcasting relay for orthogonal multiuser channels," in Proc. IEEE Global Telecommunications Conf., San Francisco, CA, Nov. 2006, pp. 1-5.

[35] M. Khojastepour and X. Wang, "Capacity bounds for MIMO shared relay channel with half-duplex constraint," in Proc. IEEE Int. Symp. Information Theory, ISIT, Jul. 6-11, 2008, pp. 1918-1922.

[36] G. Kramer and A. Van Wijngaarden, "On the white Gaussian multipleaccess relay channel," in Proc. IEEE Int. Symp. Information Theory, Jun. 25-30, 2000, p. 40.

[37] K. Azarian, H. El Gamal, and P. Schniter, "On the optimality of the ARQ-DDF protocol," IEEE Trans. Inf. Theory, vol. 54, no. 4, pp. 1718-1724, Apr. 2008.

[38] D. Chen and J. Laneman, "The diversity-multiplexing tradeoff for the multiaccess relay channel," in Proc. 40th Annu. Conf. Information Sciences and Systems, Mar. 22-24, 2006, pp. 1324-1328.

[39] D. Chen, K. Azarian, and J. N. Laneman, "A case for amplify-forward relaying in the block-fading multi-access channel," IEEE Trans. Inf. Theory, to be published.

[40] G. Kramer, P. Gupta, and M. Gastpar, "Information-theoretic multihopping for relay networks," in Proc. Int. Zurich Seminar on Communications, 2004, pp. 192-195.

[41] Y. Liang and V. V. Veeravalli, "The impact of relaying on the capacity of broadcast channels," presented at the IEEE Int. Symp. Information Theory, ISIT, Jun./Jul. 2004.

[42] Y. Jiang and V. M. K. , "Diversity-multiplexing tradeoff of MIMO system with antenna selection," in Proc. IEEE Int. Symp. Information Theory, Nice, France, June. 24-39, 2007, pp. 2836-2840.

[43] S. A. Jafar and S. Shamai, "Degrees of freedom region of the MIMO X channel," IEEE Trans. Inf. Theory, vol. 54, no. 1, pp. 151-170, Jan. 2008.

[44] M. Abouelseoud and A. Nosratina, "The gateway channel: Outage analysis," in Proc. IEEE Global Telecommunications Conf., New Orleans, LA, Dec. 2008, pp. 1-5.

[45] D. Tse and P. Viswanath, Fundamentals of Wireless Communication. Cambridge, U.K.: Cambridge Univ. Press, 2005.

[46] T. Kim and M. Skoglund, "Diversity-multiplexing tradeoff in MIMO channels with partial CSIT," IEEE Trans. Inf. Theory, vol. 53, no. 8, pp. 2743-2759, Aug. 2007. 
[47] K. Seddik, A. Sadek, W. Su, and K. Liu, "Outage analysis of multi-node amplify-and-forward relay networks," in Proc. Wireless Communications and Networking Conf., Oct. 2006, pp. 1184-1188.

Mohamed Abouelseoud (S'09) received the B.S. in electrical engineering and the M.S. in engineering mathematics from Cairo University, Egypt, in 2003 and 2006, respectively. Currently, he is working toward the Ph.D. degree in electrical engineering at the University of Texas at Dallas. His research interests lie in the broad area of wireless communication and information theory, with emphasis on cooperative communications and relay networks. Since May 2010, he has been with InterDigital Communications, Inc., King of Prussia, PA, as a Senior Systems Engineer in the Advanced Air Interface Group. He is involved in research on the next generation wireless cellular systems such as LTE-A and beyond.
Aria Nosratinia (S'87-M'97-SM'04-F'10) is the Jonsson Distinguished Professor at the Department of Electrical Engineering, the University of Texas at Dallas. He received his Ph.D. in electrical and computer engineering from the University of Illinois at Urbana-Champaign in 1996. He has held visiting appointments at Princeton University, Rice University, and UCLA. His interests lie in the broad area of information theory and signal processing, with applications in wireless communications. He is an associate editor for the IEEE TRANSACTIONS ON INFORMATION THEORY and the IEEE TRANSACTIONS ON WIRELESS COMMUNICATIONS, and was the secretary of the IEEE Information Theory Society in 2010-2011. He served as treasurer for ISIT 2010 in Austin. He has been an editor for the IEEE TRANSACTIONS ON IMAGE PROCESSING, the IEEE Signal PROCESSING LeTters, and the IEEE Wireless Communications Magazine. He has been the recipient of the National Science Foundation career award and is a Fellow of IEEE. 